\title{
ZTF Early Observations of Type Ia Supernovae. III. Early-time Colors As a Test for Explosion Models and Multiple Populations
}



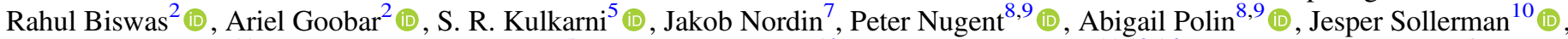 \\ Eric C. Bellm ${ }^{11}$ (1), Michael W. Coughlin ${ }^{5}$ (10), Richard Dekany ${ }^{12}$ (10), V. Zach Golkhou ${ }^{11,13,16}$ (1), Matthew J. Graham ${ }^{5}$ (1) \\ Mansi M. Kasliwal ${ }^{5}$ (1) , Thomas Kupfer ${ }^{14}$ (1), Russ R. Laher ${ }^{15}$ (1), Frank J. Masci ${ }^{15}$ (D), Michael Porter ${ }^{12}$, Ben Rusholme ${ }^{15}$ (D), and \\ David L. Shupe ${ }^{15}$ (D) \\ ${ }^{1}$ Nordita, KTH Royal Institute of Technology and Stockholm University, Roslagstullsbacken 23, SE-106 91 Stockholm, Sweden; mattia.bulla@fysik.su.se \\ ${ }^{2}$ The Oskar Klein Centre, Department of Physics, Stockholm University, AlbaNova, SE-10691 Stockholm, Sweden \\ ${ }^{3}$ Center for Interdisciplinary Exploration and Research in Astrophysics (CIERA) and Department of Physics and Astronomy, Northwestern University, \\ 2145 Sheridan Road, Evanston, IL 60208, USA \\ 4 The Adler Planetarium, Chicago, IL 60605, USA \\ ${ }^{5}$ Division of Physics, Mathematics, and Astronomy, California Institute of Technology, Pasadena, CA 91125, USA \\ ${ }^{6}$ Unidad Mixta Internacional Franco-Chilena de Astronomía, CNRS/INSU UMI 3386 and Instituto de Astrofísica, Pontificia Universidad Católica de Chile, \\ Santiago, Chile \\ ${ }^{7}$ Institute of Physics, Humboldt-Universität zu Berlin, Newtonstr. 15, D-12489 Berlin, Germany \\ ${ }^{8}$ Departments of Physics and Astronomy, University of California, Berkeley, Berkeley, CA 94720 USA \\ ${ }^{10}$ The Oskar Klein Centre, Department of Astronomy, Stockholm University, AlbaNova, SE-10691 Stockholm, Sweden \\ ${ }^{11}$ DIRAC Institute, Department of Astronomy, University of Washington, 3910 15th Avenue NE, Seattle, WA 98195, USA \\ ${ }^{12}$ Caltech Optical Observatories, California Institute of Technology, Pasadena, CA, USA \\ ${ }^{13}$ The eScience Institute, University of Washington, Seattle, WA 98195, USA \\ ${ }^{14}$ Kavli Institute for Theoretical Physics, University of California, Santa Barbara, CA 93106, USA \\ ${ }^{15}$ IPAC, California Institute of Technology, 1200 E. California Blvd, Pasadena, CA 91125, USA \\ Received 2020 January 8; revised 2020 April 14; accepted 2020 April 16; published 2020 October 9
}

\begin{abstract}
Colors of Type Ia supernovae (SNe Ia) in the first few days after explosion provide a potential discriminant between different models. In this paper, we present $g-r$ colors of $65 \mathrm{SNe}$ Ia discovered within 5 days from first light by the Zwicky Transient Facility in 2018, a sample that is about three times larger than that in the literature. We find that $g-r$ colors are intrinsically rather homogeneous at early phases, with about half of the dispersion attributable to photometric uncertainties ( $\sigma_{\text {noise }} \sim \sigma_{\text {int }} \sim 0.18 \mathrm{mag}$ ). Colors are nearly constant starting from 6 days after first light ( $g-r \sim-0.15 \mathrm{mag}$ ), while the time evolution at earlier epochs is characterized by a continuous range of slopes, from events rapidly transitioning from redder to bluer colors (slope of $\sim-0.25 \mathrm{mag} \mathrm{day}^{-1}$ ) to events with a flatter evolution. The continuum in the slope distribution is in good agreement both with models requiring some amount of ${ }^{56} \mathrm{Ni}$ mixed in the outermost regions of the ejecta and with "double-detonation" models having thin helium layers $\left(M_{\mathrm{He}}=0.01 M_{\odot}\right)$ and varying carbon-oxygen core masses. At the same time, six events show evidence for a distinctive "red bump" signature predicted by double-detonation models with larger helium masses. We finally identify a significant correlation between the early-time $g-r$ slopes and supernova brightness, with brighter events associated to flatter color evolution $(p$-value $=0.006)$. The distribution of slopes, however, is consistent with being drawn from a single population, with no evidence for two components as claimed in the literature based on $B-V$ colors.
\end{abstract}

Unified Astronomy Thesaurus concepts: Surveys (1671); Supernovae (1668); Type Ia supernovae (1728)

\section{Introduction}

Decades of observational and theoretical efforts have led to a general consensus that Type Ia supernovae (SNe Ia) arise from thermonuclear explosions of carbon-oxygen white dwarfs in binary systems. Nevertheless, the conditions leading to the thermonuclear runaway are still being debated, with the proposed scenarios typically grouped depending on whether the companion star is a nondegenerate star ("single-degenerate channel," Whelan \& Iben 1973) or another white dwarf ("double-degenerate channel," Iben \& Tutukov 1984; Webbink 1984), and whether the explosion mechanism is triggered close to the Chandrasekharmass $\left(M_{\mathrm{ch}}\right)$ limit or in a sub- $M_{\mathrm{ch}}$ white dwarf (see, e.g., Livio \& Mazzali 2018 for a recent review).

\footnotetext{
${ }^{16}$ Moore-Sloan, WRF Innovation in Data Science, and DIRAC Fellow.
}

Colors of SNe Ia are controlled by the interplay between cooling from the ejecta expansion and heating due to thermalization of gamma rays from the decay of radioactive elements (but are also affected by composition and line blanketing effects). Especially at early times, the color evolution can be used to probe the location within the ejecta of ${ }^{56} \mathrm{Ni}$ and other radioactive isotopes (Dessart et al. 2014) and help discriminate between different models. For instance, models producing ${ }^{56} \mathrm{Ni}$ in the high-density innermost regions of the ejecta are expected to have red colors early on-when the relatively cold outer ejecta are probed-while showing a transition to bluer colors with the photosphere receding into increasingly hotter layers. In contrast, models with radioactive material mixed in the outer ejecta will be relatively bluer at early phases due to the additional source of heating from radioactive decay. 
An interesting example in this respect is the so-called sub- $M_{\text {ch }}$ "double-detonation" scenario, where a first detonation in a thin helium layer accreted on the surface triggers a second detonation in the carbon-oxygen core (e.g., Nomoto 1980; Taam 1980; Livne 1990; Fink et al. 2010; Moll \& Woosley 2013). Radioactive elements are produced both in a thin outermost layer and in the inner regions. These two distinct radioactive sources lead to blue colors at different times (soon after explosion and a few days later, respectively), with the transition in between producing a distinctive signature at early times, dubbed "red bump" (Noebauer et al. 2017; Maeda et al. 2018; Polin et al. 2019a). The double-detonation mechanism has been invoked to explain three recent supernova Type Ia (SN Ia) events (Jiang et al. 2017; De et al. 2019; JacobsonGalan et al. 2020). Other interesting scenarios involving the interaction of $\mathrm{SN}$ ejecta with either a nondegenerate companion star (Kasen 2010) or unbound material ejected prior to detonation (pulsational-delayed-detonation (PDDEL) models, Dessart et al. 2014) predict rather blue colors soon after explosion ( $g-r \lesssim 0$ mag).

Early-time observations of SNe Ia are challenging and thus have historically been limited to very nearby events. Stritzinger et al. (2018) presented a sample of $13 \mathrm{SNe}$ Ia with colors at epochs earlier than 5 days from inferred first light. Based on the $B-V$ color evolution in the first $\sim 5$ days, they claim evidence for two distinct populations, with a so-called "red" class showing a steep transition from red to bluer colors and a "blue" class characterized by bluer colors and flatter evolution. They suggested that events in the blue class are preferentially overluminous and of the Branch Shallow Silicon spectral type, while those in the red class are more typically associated with the Branch Core-Normal or Cool type (Branch et al. 2006). Similar conclusions were drawn by Jiang et al. (2018) when inspecting light curves of 23 relatively young SNe Ia. Recently, Han et al. (2020) added six events to the sample of Stritzinger et al. (2018) and claimed to confirm the distinction between red and blue events (but see discussion in Section 5).

Thanks to the advent of wide-field optical surveys, discovering $\mathrm{SNe}$ Ia in their infancy has now become easier (e.g., Hosseinzadeh et al. 2017; Miller et al. 2018; Dimitriadis et al. 2019; Li et al. 2019; Papadogiannakis et al. 2019; Shappee et al. 2019; Vallely et al. 2019). As the final in a series of three papers, here we report colors of 65 SNe Ia discovered within 5 days from inferred first light by the Zwicky Transient Facility (ZTF; Bellm et al. 2019; Graham et al. 2019; Masci et al. 2019) in 2018, a sample that to date is about three times larger than that available in the literature. ${ }^{17}$ In particular, we study the $g-r$ color evolution of our sample to place constraints on explosion models and at the same time test claims of two distinct populations in the early-time colors. Details of the sample are discussed in Yao et al. (2019), while the analysis of $g$ and $r$ light curves is presented in Miller et al. (2020).

The paper is organized as follows. We provide details of the sample selection and of the analysis in Section 2, while presenting the inferred $g-r$ colors in Section 3. We then compare our data to models in Section 4 and test the presence

\footnotetext{
17 Here we count only events with the first color measurement within 5 days from first light, i.e., a total of 19 SNe combining the sample of Stritzinger et al. (2018) and Han et al. (2020). The sample of Jiang et al. (2018) reports discovery phases relative to maximum light rather than first light (see their Table 1).
}

of multiple populations in Section 5. We finally discuss our results and draw conclusions in Section 6.

\section{Data Sample}

For our study, we use high-quality $g_{\text {ZTF }}$ and $r_{\mathrm{ZTF}}$ (hereafter $g$ and $r$ ) light curves of SNe Ia discovered by the ZTF in 2018. Details of the sample selection are discussed in Yao et al. (2019, see their Table 2). Briefly, 247 spectroscopically classified SNe Ia were found by the high-cadence (six epochs per night, $3 g+3 r)$ ZTF partnership survey in 2018. Among these, $127 \mathrm{SNe}$ were discovered earlier than -10 days (in rest frame) relative to $B$-band peak brightness. Forced-PSF (Point Spread Function) photometry performed by Yao et al. (2019) is used in this work for all the $\mathrm{SNe}$ in the sample. Following suggestions from Yao et al. (2019, see their section 3.5), we remove observations with either high reduced chi-square statistics $\left(\chi_{\nu}^{2}>4\right)$ or large baseline offset $C(|C|>15)$. This cut reduces the sample to 94 events.

In this paper, we are interested in studying colors of $\mathrm{SNe}$ Ia during the early phases following the explosion. As in Stritzinger et al. (2018), we choose to describe the color evolution of $\mathrm{SNe}$ in our sample with respect to the first-light epoch $t_{\mathrm{fl}}$, inferred by simultaneously fitting the early-time flux in both $g\left(f_{g}\right)$ and $r\left(f_{r}\right)$ band

$$
f_{i}(t)=C+H\left[t_{\mathrm{fl}}\right] A_{i}\left(t-t_{\mathrm{fl}}\right)^{\alpha_{i}} \quad i=g, r,
$$

where $A_{i}$ is a scale factor, $t$ is the time, $\alpha_{i}$ is a power-law index, and $H\left[t_{\mathrm{fl}}\right]$ is the Heaviside step function $\left(H=0\right.$ for $t<t_{\mathrm{fl}}$ and $H=1$ otherwise). In this work, we adopt first-light epochs $t_{\mathrm{fl}}$ from Miller et al. (2020), which reported values for two different set of models: one where an uninformative prior is assumed for $\alpha_{i}$ and one where $\alpha_{g}=\alpha_{r}=2$ (i.e., the $t^{2}$ model widely used in the literature, also known as the "fireball" model, Riess et al. 1999). For each SN, we use the Deviance Information Criterion (Spiegelhalter et al. 2002) to choose what model better describes the early light curve and thus to select the corresponding $t_{\mathrm{fl}}$ value (see Miller et al. 2020 for more details).

Here, we adopt the same cut made by Stritzinger et al. (2018) and restrict to $\mathrm{SNe}$ that have the first color measurement within 5 days from $t_{\mathrm{fl}}$. This leads to a sample of $65 \mathrm{SNe} \mathrm{Ia}$, which comprises 56 normal SNe Ia, six over-luminous (91T-/99aalike) $\mathrm{SNe} \mathrm{Ia}$, one "super-Chandrasekhar" $\mathrm{SN}$, one "Ia-CSM" $\mathrm{SN}$, and one "02cx-like" SN according to the spectroscopic classification in Yao et al. (2019). Table 1 provides information about the $65 \mathrm{SNe}$ Ia. As expected, SNe at higher compared to lower redshifts are discovered relatively later in their evolution. Specifically, the 21 events at $z \gtrsim 0.08$ are all discovered in both $g$ and $r$ filters later than 2.5 days after $t_{\mathrm{fl}}$.

In order to decrease the uncertainties on each data point, we average observations within the same night and then select $3 \sigma$ detections for our analysis. We then calculate $g-r$ colors for nights with detections in both $g$ and $r$. The following corrections are applied to $g$ and $r$ photometry before calculating the $g-r$ colors: (i) time-dilation correction; (ii) Milky Way reddening correction; (iii) host-galaxy reddening correction; (iv) $K$-correction. Redshift and $E(B-V)_{\mathrm{MW}}$ values from Table 3 of Yao et al. (2019) are used for step (i) and (ii), while the full light curves ${ }^{18}$ are fit using the program SNoOPY

\footnotetext{
${ }^{18} \mathrm{SNe}$ in our sample are observed for a median of $\sim 80 \%$ of the nights in the first 30 days since discovery (see Yao et al. 2019 for more details on the lightcurve sampling).
} 
Table 1

Properties of the $65 \mathrm{SNe}$ Ia in Our Sample

\begin{tabular}{|c|c|c|c|c|c|c|c|c|}
\hline ZTF Name & $\begin{array}{l}\text { TNS Name } \\
\text { (2) }\end{array}$ & $\begin{array}{l}\text { Ia Subtype } \\
\text { (3) }\end{array}$ & $\begin{array}{l}\text { Redshift } \\
\text { (days) } \\
\text { (4) }\end{array}$ & $\begin{array}{c}t_{\mathrm{g}-\mathrm{r}}^{\text {first }}-t_{\mathrm{fl}} \\
\left(\mathrm{mag} \mathrm{day}^{-1}\right) \\
(5)\end{array}$ & $\begin{array}{c}\Delta(g-r) / \Delta t \\
\text { (6) }\end{array}$ & $\begin{array}{c}\text { SALT2 } x_{1} \\
(\mathrm{mag}) \\
(7)\end{array}$ & $\begin{array}{c}E(B-V)_{\text {host }} \\
(\mathrm{mag}) \\
(8)\end{array}$ & $\begin{array}{c}\left.K_{\mathrm{gr}}\right\rangle \\
(9)\end{array}$ \\
\hline ZTF 18aapqwyv & SN 2018bhc & Normal* & 0.0560 & $2.11_{-0.69}^{+0.53}$ & $-0.16 \pm 0.16$ & $-1.72 \pm 0.18$ & 0.259 & $\overline{0.042}$ \\
\hline ZTF 18aapsedq & SN 2018bgs & Normal ${ }^{*}$ & 0.0720 & $3.72_{-0.31}^{+0.31}$ & $\cdots$ & $-0.09 \pm 0.18$ & 0.011 & 0.073 \\
\hline ZTF 18aaqcugm & SN 2018bhi & Normal & 0.0619 & $4.50_{-0.24}^{+0.22}$ & $\cdots$ & $-1.12 \pm 0.12$ & 0.005 & 0.050 \\
\hline ZTF 18aaqqoqs & SN 2018cbh & 99aa-like & 0.082 & $3.30_{-0.23}^{+0.23}$ & $\ldots$ & $1.22 \pm 0.27$ & 0.044 & 0.083 \\
\hline ZTF 18aarldnh & SN 2018lpd & Normal & 0.1077 & $3.84_{-0.64}^{+0.57}$ & $-0.28 \pm 0.25$ & $-1.05 \pm 0.38$ & 0.141 & 0.065 \\
\hline ZTF 18aasdted & SN 2018big & Normal & 0.0181 & $1.25_{-0.10}^{+0.09}$ & $\ldots$ & $0.85 \pm 0.05$ & 0.257 & -0.001 \\
\hline ZTF 18aaslhxt & SN 2018btk & Normal & 0.0551 & $2.15_{-0.09}^{+0.09}$ & $\ldots$ & $0.29 \pm 0.02$ & 0.000 & 0.039 \\
\hline ZTF 18aaumlfl & SN 2018btg & Normal & 0.0874 & $4.13_{-0.40}^{+0.37}$ & $\cdots$ & $-1.13 \pm 0.26$ & 0.027 & 0.070 \\
\hline ZTF 18aauocnw & SN 2018cae & Normal & 0.102 & $3.22_{-0.57}^{+0.50}$ & $-0.05 \pm 0.14$ & $0.14 \pm 0.27$ & 0.131 & 0.088 \\
\hline ZTF 18aavrwhu & SN 2018bxo & Normal & 0.0620 & $4.62_{-0.22}^{+0.21}$ & $\ldots$ & $1.20 \pm 0.27$ & 0.044 & 0.060 \\
\hline ZTF 18aaxcntm & SN 2018ccl & normal & 0.0269 & $3.78_{-0.15}^{+0.15}$ & $\ldots$ & $-1.52 \pm 0.06$ & 0.213 & 0.012 \\
\hline ZTF 18aaxdrjn & SN 2018cdt & Normal & 0.0340 & $4.47_{-0.13}^{+0.13}$ & $\cdots$ & $-1.92 \pm 0.09$ & 0.000 & 0.021 \\
\hline ZTF 18aaxqyki & SN $2018 \mathrm{cnz}$ & Normal & 0.1003 & $3.70_{-0.54}^{+0.49}$ & $\ldots$ & $0.94 \pm 0.27$ & 0.025 & 0.075 \\
\hline ZTF 18aaxsioa & SN 2018cfa & Normal ${ }^{*}$ & 0.0315 & $3.39_{-0.07}^{+0.07}$ & $0.00 \pm 0.02$ & $-1.51 \pm 0.06$ & 0.150 & 0.013 \\
\hline ZTF 18aaxvpsw & SN 2018cof & Normal & 0.0916 & $4.10_{-0.56}^{+0.48}$ & $-0.07 \pm 0.12$ & $0.04 \pm 0.35$ & 0.083 & 0.069 \\
\hline ZTF 18aaxwjmp & SN 2018coe & Normal & 0.084 & $4.04_{-0.21}^{+0.20}$ & $-0.03 \pm 0.13$ & $0.42 \pm 0.15$ & 0.100 & 0.081 \\
\hline ZTF 18aayjvve & SN 2018cny & Normal & 0.0474 & $2.19_{-0.43}^{+0.38}$ & $-0.13 \pm 0.02$ & $-0.09 \pm 0.10$ & 0.164 & 0.036 \\
\hline ZTF 18aaykjei & SN 2018crl & Ia-CSM & 0.0970 & $4.33_{-0.31}^{+0.30}$ & $\ldots$ & $4.14 \pm 0.21$ & 0.000 & 0.090 \\
\hline ZTF 18aazblzy & SN 2018cri & Normal & 0.0653 & $1.36_{-0.09}^{+0.08}$ & $-0.06 \pm 0.06$ & $-1.68 \pm 0.09$ & 0.016 & 0.054 \\
\hline ZTF 18aazixbw & SN 2018coi & Normal & 0.0594 & $2.67_{-0.14}^{+0.13}$ & $-0.05 \pm 0.10$ & $-1.58 \pm 0.13$ & 0.147 & 0.054 \\
\hline ZTF 18aazsabq & SN 2018crn & Normal & 0.060 & $2.71_{-0.63}^{+0.53}$ & $-0.15 \pm 0.01$ & $-1.24 \pm 0.12$ & 0.123 & 0.044 \\
\hline ZTF 18abatffv & SN 20181pf & Normal & 0.143 & $4.45_{-0.64}^{+0.57}$ & $\cdots$ & $0.95 \pm 0.56$ & 0.117 & 0.099 \\
\hline ZTF 18abauprj & SN 2018cnw & 99aa-like & 0.0242 & $1.38_{-0.10}^{+0.10}$ & $-0.05 \pm 0.02$ & $1.34 \pm 0.04$ & 0.029 & -0.003 \\
\hline ZTF 18abaxlpi & SN $2018 \mathrm{ctm}$ & Normal & 0.0642 & $1.64_{-0.17}^{+0.17}$ & $-0.04 \pm 0.04$ & $0.14 \pm 0.20$ & 0.160 & 0.051 \\
\hline ZTF 18abcflnz & SN 2018cuw & Normal & 0.0273 & $2.82_{-0.22}^{+0.20}$ & $-0.12 \pm 0.04$ & $0.11 \pm 0.02$ & 0.050 & 0.000 \\
\hline ZTF 18abckujg & SN 2018cvt & Normal & 0.075 & $2.68_{-0.25}^{+0.26}$ & $\cdots$ & $0.50 \pm 0.30$ & 0.078 & 0.062 \\
\hline ZTF 18abckujq & SN 2018cvf & Normal & 0.0638 & $3.07_{-0.40}^{+0.37}$ & $\ldots$ & $0.51 \pm 0.39$ & 0.008 & 0.058 \\
\hline ZTF 18abclfee & SN 2018cxk & 02cx-like & 0.0290 & $0.46_{-0.16}^{+0.12}$ & $0.02 \pm 0.01$ & $-2.53 \pm 0.09$ & 0.087 & 0.024 \\
\hline ZTF 18abcrxoj & SN 2018cvw & Normal & 0.0309 & $0.98_{-0.07}^{+0.07}$ & $-0.02 \pm 0.03$ & $-1.29 \pm 0.06$ & 0.161 & 0.013 \\
\hline ZTF 18abdbuty & SN 2018dbd & Normal & 0.059 & $2.65_{-0.26}^{+0.25}$ & $-0.06 \pm 0.03$ & $-0.76 \pm 0.31$ & 0.138 & 0.047 \\
\hline ZTF 18abdefet & SN 2018dds & Normal & 0.074 & $3.66_{-0.81}^{+0.70}$ & $0.11 \pm 0.06$ & $-0.12 \pm 0.31$ & 0.265 & 0.064 \\
\hline ZTF 18abdfydj & SN 2018dzr & Normal & 0.076 & $3.99_{-0.30}^{+0.30}$ & $\cdots$ & $0.24 \pm 0.26$ & 0.054 & 0.079 \\
\hline ZTF 18abdkimx & SN 2018dyq & Normal & 0.077 & $4.00_{-0.46}^{+0.43}$ & $\cdots$ & $-0.05 \pm 0.05$ & 0.079 & 0.078 \\
\hline ZTF 18abdpvnd & SN 2018dvf & $\mathrm{SC}$ & 0.050 & $3.58_{-0.21}^{+0.20}$ & $\ldots$ & $3.06 \pm 0.10$ & 0.074 & 0.030 \\
\hline ZTF 18abeecwe & SN 2018dje & Normal & 0.0393 & $2.13_{-0.09}^{+0.09}$ & $-0.06 \pm 0.04$ & $-0.56 \pm 0.11$ & 0.135 & 0.015 \\
\hline ZTF 18abeegsl & SN 2018eag & Normal & 0.072 & $4.12_{-0.40}^{+0.37}$ & $\ldots$ & $-2.20 \pm 0.19$ & 0.109 & 0.056 \\
\hline ZTF 18abetehf & SN 2018dvb & Normal & 0.0649 & $2.89_{-0.14}^{+0.14}$ & $-0.01 \pm 0.09$ & $-1.37 \pm 0.23$ & 0.000 & 0.057 \\
\hline ZTF 18abfgygp & SN 2018ead & Normal & 0.064 & $2.66_{-0.53}^{+0.45}$ & $-0.02 \pm 0.05$ & $0.08 \pm 0.02$ & 0.037 & 0.059 \\
\hline ZTF 18abfhaji & SN 2018dsw & Normal & 0.084 & $2.90_{-0.20}^{+0.19}$ & $0.02 \pm 0.12$ & $-0.19 \pm 0.04$ & 0.056 & 0.072 \\
\hline ZTF 18abfhryc & SN 2018dhw & Normal & 0.0323 & $4.21_{-0.59}^{+0.53}$ & $\cdots$ & $0.47 \pm 0.04$ & 0.084 & 0.003 \\
\hline ZTF 18abfwuwn & SN 2018ecq & 99aa-like* & 0.109 & $4.38_{-0.22}^{+0.21}$ & $\ldots$ & $0.60 \pm 0.30$ & 0.059 & 0.080 \\
\hline ZTF 18abgmcmv & SN 2018eay & 91T-like & 0.0185 & $1.20_{-0.15}^{+0.14}$ & $-0.06 \pm 0.01$ & $0.69 \pm 0.05$ & 0.770 & -0.009 \\
\hline ZTF 18abgxvra & SN 2018efb & Normal & 0.104 & $3.14_{-0.20}^{+0.19}$ & $0.12 \pm 0.07$ & $0.80 \pm 0.24$ & 0.003 & 0.060 \\
\hline ZTF 18abimsyv & SN 2018eni & Normal ${ }^{*}$ & 0.088 & $2.71_{-0.15}^{+0.14}$ & $0.02 \pm 0.06$ & $1.05 \pm 0.16$ & 0.033 & 0.083 \\
\hline ZTF 18abjtger & SN 2018err & Normal & 0.107 & $4.79_{-1.00}^{+0.91}$ & $\ldots$ & $0.77 \pm 0.66$ & 0.069 & 0.071 \\
\hline ZTF 18abjvhec & SN 2018emv & Normal & 0.0570 & $3.39_{-0.31}^{+0.31}$ & $\cdots$ & $0.37 \pm 0.41$ & 0.052 & 0.052 \\
\hline ZTF 18abkhcrj & SN 2018emi & Normal & 0.0383 & $3.68_{-0.22}^{+0.21}$ & $-0.05 \pm 0.05$ & $0.85 \pm 0.16$ & 0.310 & 0.022 \\
\hline ZTF 18abkhcwl & SN 2018eml & Normal & 0.0317 & $3.46_{-0.43}^{+0.39}$ & $\ldots$ & $0.08 \pm 0.09$ & 0.027 & 0.014 \\
\hline ZTF 18abkhdxe & SN 2018ffg & Normal & 0.104 & $4.55_{-0.67}^{+0.62}$ & $\ldots$ & $0.73 \pm 0.43$ & 0.167 & 0.056 \\
\hline ZTF 18abmmkaz & SN 2018fdz & 99aa-like* & 0.063 & $4.78_{-2.87}^{+3.12}$ & $\ldots$ & $0.74 \pm 0.15$ & 0.028 & 0.047 \\
\hline ZTF 18abmxdhb & SN 2018fjv & Normal & 0.070 & $4.96_{-0.43}^{+0.42}$ & $\cdots$ & $1.27 \pm 0.22$ & 0.026 & 0.056 \\
\hline ZTF 18abokpvh & SN 2018fnc & Normal* & 0.081 & $3.41_{-0.21}^{+0.20}$ & $0.04 \pm 0.06$ & $0.77 \pm 0.22$ & 0.000 & 0.057 \\
\hline ZTF 18abpamut & SN 2018fqe & Normal ${ }^{*}$ & 0.064 & $1.00_{-0.51}^{+0.37}$ & $-0.03 \pm 0.00$ & $0.83 \pm 0.29$ & 0.185 & 0.061 \\
\hline ZTF 18abpaywm & SN 2018fne & Normal & 0.040 & $1.70_{-0.14}^{+0.14}$ & $0.11 \pm 0.10$ & $0.61 \pm 0.14$ & 0.309 & 0.019 \\
\hline ZTF 18abpmmpo & SN 2018fnd & 99aa-like & 0.076 & $3.98_{-0.25}^{+0.23}$ & $\cdots$ & $1.50 \pm 0.27$ & 0.034 & 0.064 \\
\hline ZTF 18abpttky & SN 2018fse & Normal & 0.084 & $3.92_{-0.51}^{+0.45}$ & $\cdots$ & $-1.31 \pm 0.40$ & 0.073 & 0.066 \\
\hline
\end{tabular}


Table 1

(Continued)

\begin{tabular}{|c|c|c|c|c|c|c|c|c|}
\hline ZTF Name & $\begin{array}{l}\text { TNS Name } \\
\text { (2) }\end{array}$ & $\begin{array}{l}\text { Ia Subtype } \\
\text { (3) }\end{array}$ & $\begin{array}{l}\text { Redshift } \\
\text { (days) } \\
\text { (4) }\end{array}$ & $\begin{array}{c}t_{\mathrm{g}-\mathrm{r}}^{\text {first }}-t_{\mathrm{fl}} \\
\left(\mathrm{mag} \mathrm{day}^{-1}\right) \\
(5)\end{array}$ & $\begin{array}{c}\Delta(g-r) / \Delta t \\
(6)\end{array}$ & $\begin{array}{c}\text { SALT2 } x_{1} \\
(\mathrm{mag}) \\
(7)\end{array}$ & $\begin{array}{c}E(B-V)_{\text {host }} \\
(\mathrm{mag}) \\
(8)\end{array}$ & $\begin{array}{c}\left\langle K_{\mathrm{gr}}\right\rangle \\
(9)\end{array}$ \\
\hline ZTF 18absdgon & SN 2018frx & Normal $^{*}$ & 0.0620 & $3.63_{-0.21}^{+0.20}$ & $-0.08 \pm 0.06$ & $-0.26 \pm 0.18$ & 0.295 & 0.048 \\
\hline ZTF 18abukmty & SN 2018lpz & Normal $^{*}$ & 0.104 & $3.76_{-0.42}^{+0.39}$ & $0.23 \pm 0.25$ & $0.51 \pm 0.32$ & 0.085 & 0.066 \\
\hline ZTF 18abvbayb & SN 2018lpq & Normal & 0.132 & $3.36_{-0.36}^{+0.34}$ & $0.00 \pm 0.14$ & $-0.20 \pm 0.31$ & 0.048 & 0.056 \\
\hline ZTF $18 \mathrm{abwdcdv}$ & SN 2018gre & Normal & 0.0538 & $2.50_{-0.17}^{+0.17}$ & $-0.07 \pm 0.11$ & $-0.46 \pm 0.12$ & 0.457 & 0.042 \\
\hline ZTF 18abwtops & SN 2018lqa & Normal & 0.101 & $3.78_{-0.36}^{+0.34}$ & $-0.21 \pm 0.02$ & $-1.38 \pm 0.28$ & 0.015 & 0.055 \\
\hline ZTF 18abxxssh & SN 2018gvj & Normal & 0.0782 & $3.52_{-0.21}^{+0.20}$ & ... & $1.53 \pm 0.24$ & 0.000 & 0.061 \\
\hline ZTF 18abxygvv & SN 2018gwb & Normal $^{*}$ & 0.079 & $1.63_{-0.16}^{+0.15}$ & $-0.07 \pm 0.11$ & $-0.10 \pm 0.22$ & 0.020 & 0.057 \\
\hline
\end{tabular}

Note. Column (3): classification from Yao et al. (2019), ending with an asterisk in cases where classification could not be reliably determined from spectroscopy alone Column (4): redshift from Yao et al. (2019), shown with three decimals when inferred from the SNID fit of SN spectra and with four decimals otherwise. Column (5): rest-frame time of first detection in both $g$ and $r$ relative to first light $t_{\mathrm{fl}}$. Column (6): $g-r$ linear slope in the first 6 days for the $35 \mathrm{SNe}$ with at least three data points in this time window. Column (7): SALT2 $x_{1}$ parameter from Yao et al. (2019). Column (8): host reddening inferred using SNooPy (Burns et al. 2014). Column (9): averaged $K$-correction in the first 5 days since $t_{\mathrm{fl}}$ inferred using SNooPy (Burns et al. 2014).

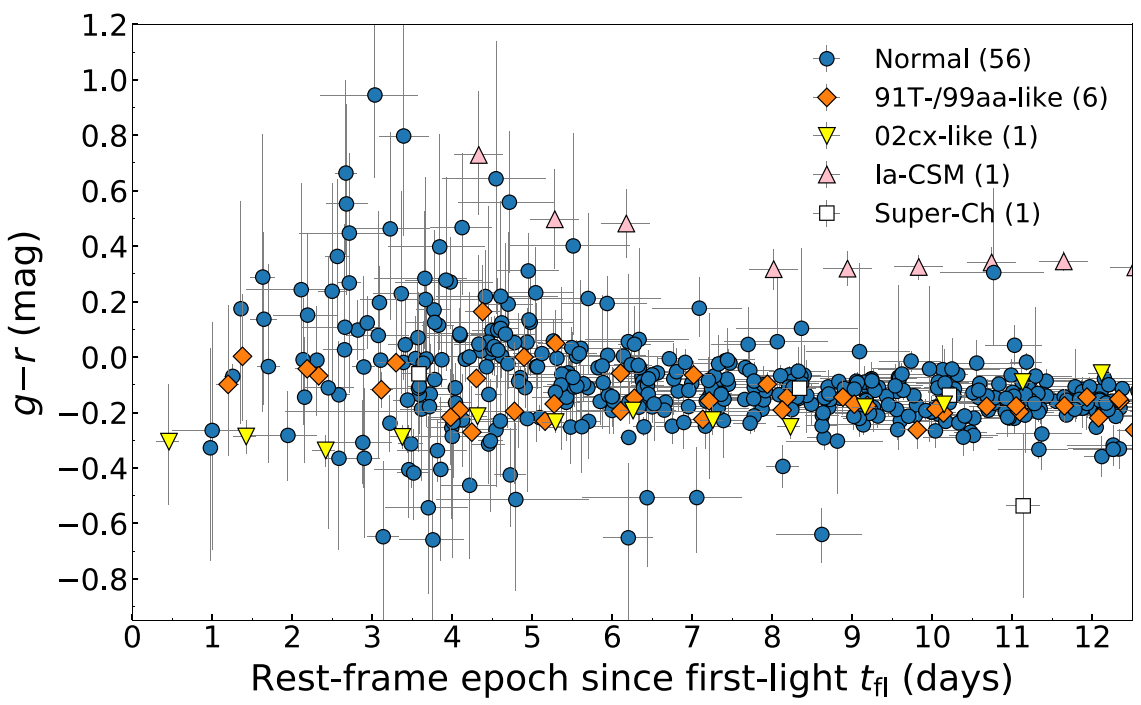

Figure 1. Evolution of $g-r$ colors for the $65 \mathrm{SNe}$ Ia discovered by ZTF within 5 days from first light $t_{\mathrm{ff}}$. The sample includes 56 spectroscopically normal SNe Ia (blue circles), six over-luminous 91T-/99aa-like SNe Ia (orange diamonds), one "02cx-like" SN (yellow triangles down), one "Ia-CSM" SN (pink triangle up) and one "super-Chandrasekhar" SN (white squares). Colors are corrected for reddening (both Milky Way and host) and $K$-correction.

(Burns et al. 2014) to infer $E(B-V)_{\text {host }}$ and $K$-correction values for step (iii) and (iv). Host reddening and $K$-correction values are reported for each $\mathrm{SN}$ in Table 1.

The samples of Stritzinger et al. (2018) and Han et al. (2020) include only low-redshift SNe $(0.001 \lesssim z \lesssim 0.023)$, while our sample extends to higher redshifts $(0.018 \lesssim z \lesssim 0.143)$ and it thus requires $K$-corrections. We note that $K$-corrections are not well known in the first few days following the explosion. In particular, SNooPy estimates $K$-corrections by adopting the spectral template from Hsiao et al. (2007), defined from 15 days before peak, and using an extrapolation at earlier epochs. Nevertheless, we find in Section 3.1 that our $g-r$ colors agree well with those from the low-redshift sample of Stritzinger et al. (2018), thus giving us confidence about the $K$ corrections applied to our sample. In addition, we will base most of the discussion on the time evolution (Section 3.2) rather than the absolute values (Section 3.1) of colors as this choice is less sensitive to uncertainties on $K$-corrections.

\section{Results}

In the following, we present our results and discuss the inferred colors (Section 3.1) and color evolution (Section 3.2) for the $65 \mathrm{SNe}$ Ia in our sample.

\subsection{Colors}

Figure 1 shows the $g-r$ colors for the sample of $65 \mathrm{SNe}$ Ia discovered by ZTF within 5 days from first light $t_{\mathrm{fl}}$. The distribution of $g-r$ values is rather homogeneous starting from about 6 days after $t_{\mathrm{fl}}$, with colors clustering around $g-r \sim-0.15$ mag. ${ }^{19}$ In contrast, the scatter is found to be larger at earlier epochs. However, some fraction of the scatter observed at very early times is caused by the relatively high

\footnotetext{
19 The peculiar "Ia-CSM" SN ZTF 18aaykjei (SN 2018crl) is characterized by redder colors $\left(g-r \sim 0.3 \mathrm{mag}\right.$ ) due to $\mathrm{H}_{\alpha}$ emission at wavelengths covered by the ZTF $r$ filter.
} 


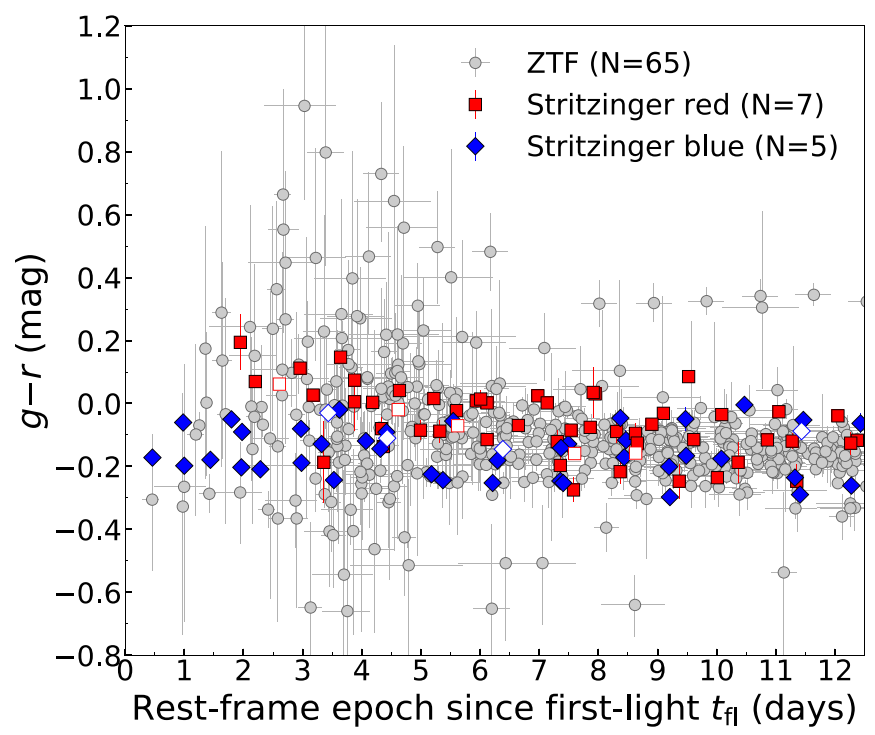

Figure 2. $g-r$ color evolution of our ZTF sample (gray circles), compared to that of 12 SNe Ia from Stritzinger et al. (2018), which has available $g$ and $r$ photometry (filled symbols, Graham et al. 2015, 2017; Hsiao et al. 2015; Shappee et al. 2016; Hosseinzadeh et al. 2017; Burns et al. 2018; Miller et al. 2018; Vinkó et al. 2018) or early-time spectra (open symbols, Foley et al. 2012; Silverman et al. 2012). Following Stritzinger et al. (2018), the 12 $\mathrm{SNe}$ are divided in red (red squares) and blue (blue diamonds) objects. Time of first light and reddening values are taken from Stritzinger et al. (2018).

photometric uncertainties that characterize most of our $\mathrm{SNe}$ when first detected. In particular, the typical uncertainties at these early epochs have a median value of $\sigma_{\text {noise }} \sim 0.18 \mathrm{mag}$, while the $g-r$ distribution in the first 6 days after $t_{\mathrm{fl}}$ has a width of $\sigma=0.23 \mathrm{mag}$. Following the light-curve rise and corresponding increase in signal to noise, both the uncertainties and the scatter in colors decrease, with the latter always $\sim 40 \%$ larger than the former. Based on these numbers, we conclude that roughly half of the scatter observed in our colors at early times ( $\lesssim 10$ days) is intrinsic and half is due to photometric uncertainties, i.e., $\sigma_{\text {int }} \sim \sigma_{\text {noise }} \sim \sigma / \sqrt{2}$. The fact that $\sigma_{\text {int }} \lesssim$ 0.18 mag in the first 6 days after first light suggests that SNe Ia are intrinsically more homogeneous in $g-r$ compared to what has been found in $B-V$ colors (Stritzinger et al. 2018). This is in qualitative agreement with the finding in Miller et al. (2020); see their Section 4.3.

The larger homogeneity of $g-r$ relative to $B-V$ colors is confirmed when comparing our sample to the $12 \mathrm{SNe}$ Ia of Stritzinger et al. (2018), which has available $g$ and $r$ photometry (Graham et al. 2015, 2017; Hsiao et al. 2015; Shappee et al. 2016; Hosseinzadeh et al. 2017; Burns et al. 2018; Miller et al. 2018; Vinkó et al. 2018) or early-time spectra to compute synthetic photometry (Foley et al. 2012; Silverman et al. 2012). As shown in Figure 2, no clear gap is found in $g-r$ at early phases between the red and blue class introduced in $B-V$ colors by Stritzinger et al. (2018), corroborating the idea that the early-time color evolution in SNe Ia might be rather homogeneous in $g$ and $r$ filters. Figure 2 also highlights how the color evolution of the ZTF sample is consistent with that reported by Stritzinger et al. (2018). The good agreement between the two samples gives us confidence about both the extinction- and $K$-corrections applied to our sample.

As shown in Figure 3, the larger homogeneity in $g-r$ compared to the $B-V$ colors can be understood as a

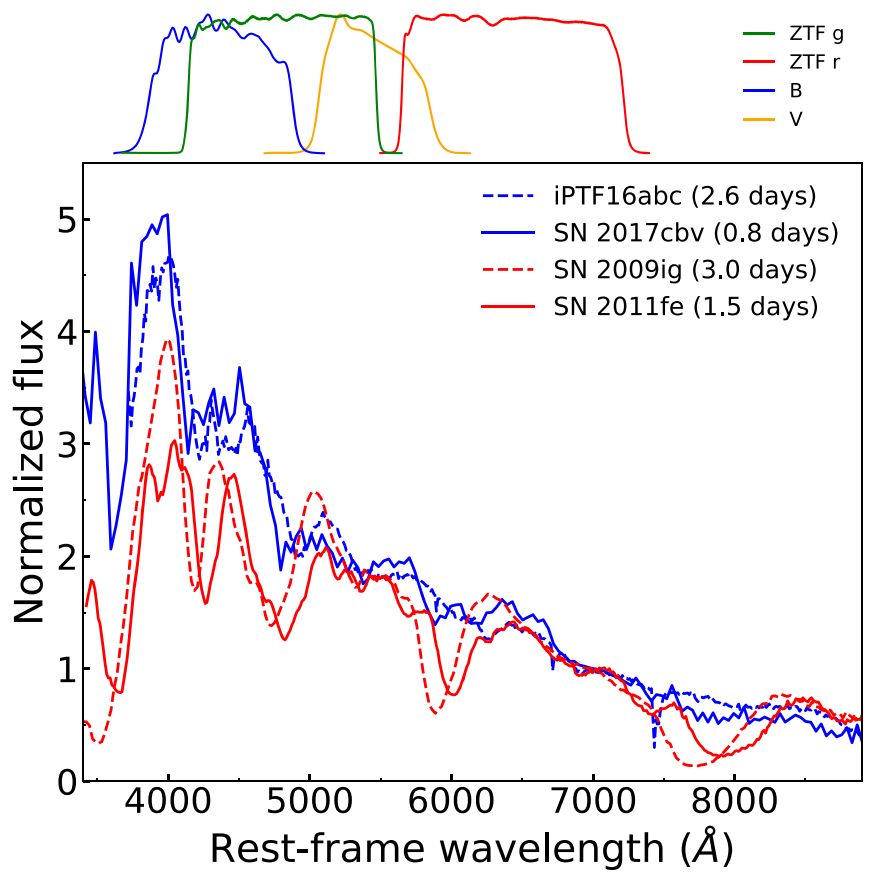

Figure 3. Early-time spectra of iPTF16abc (Miller et al. 2018), SN 2017cbv (Hosseinzadeh et al. 2017), SN 2009ig (Foley et al. 2012), and SN 2011fe (Nugent et al. 2011). ZTF $g$ and $r$ filters are shown at the top together with $B$ and $V$ filters. Spectra have been normalized at $7000 \AA$ and rebinned for presentation purpose.

consequence of the different parts of the spectral energy distribution probed by different filter combinations. Early-time spectra of four SNe Ia in the Stritzinger et al. (2018) sample are shown, where two events (iPTF16abc and SN 2017cbv) are from the so-called blue class and two (SN 2009ig and SN $2011 \mathrm{fe}$ ) are from the red class. In the wavelength region probed by the four filters, the largest spectral diversities between the two classes are seen at wavelengths below $\sim 4800 \AA$ and around the Si II $\lambda 6355$. These follow from blue objects being 91T-/99aa-like SNe, events that have been shown (e.g., Jeffery et al. 1992; Ruiz-Lapuente et al. 1992; Mazzali et al. 1995) to be more highly ionized than normal SNe Ia and thus lack singly ionized absorption features such as Si II $\lambda 6355$ at these early phases. The ZTF $g$ and $r$ filters are broader than the $B$ and $V$ filters and cover both regions with large spectral diversity. In contrast, while the $B$ filter probes the region below $\sim 4800 \AA$, the $V$ filter covers a region around $5000 \AA$ that is relatively homogeneous between the two classes. In addition, the $B$ filter extends to bluer wavelengths than the $g$ filter, in a spectral range $(\sim 3800-4200 \AA)$ with pronounced differences between blue and red objects. Therefore, the largest contrast between the two classes is captured by $B-V$ colors, while $g-r$ colors tend to wash out the observed spectral differences (this is similar to what is found at later epochs by Nordin et al. 2018, see top panel of their Figure 3). This comparison explains why $g-r$ colors are found to be more homogeneous than $B-V$ in the first few days after explosion. At the same time, it suggests that $B$ and $V$ filters might be the better choice to test different models affecting the early-time colors.

\subsection{Color Evolution}

Despite the homogeneity of $g-r$ values discussed above, we do see a distinct color evolution. Figure 4 shows the $g-r$ color evolution of each individual $\mathrm{SN}$ in our sample. To 
characterize the change in colors we restrict ourselves to events that have at least three data points in the first 6 days, resulting in a sample of $35 \mathrm{SNe} \mathrm{Ia}$. We then characterize the color evolution by performing a weighted least-square linear fit to $g-r$ in the first 6 days and infer a slope $\Delta(g-r) / \Delta t$ for each $\mathrm{SN}$, with positive (negative) values associated to colors becoming redder (bluer). Results of these fits are shown in Figure 4 and reported in Table 1 for the $35 \mathrm{SNe}$ Ia that meet the criteria defined above.

As shown in Figure 4, some events are characterized by a clear transition from redder to bluer colors and thus a negative slope, $\Delta(g-r) / \Delta t<0$, others by a flatter evolution, $\Delta(g-$ $r) / \Delta t \sim 0$. We note that all the three over-luminous 91T-/99aa-like $\mathrm{SNe}$ are characterized by relatively flat color evolutions, in agreement with findings from Stritzinger et al. (2018). The full range of slopes, going from a minimum of $\Delta(g-r) / \Delta t \sim-0.28$ to a maximum of $\Delta(g-r) / \Delta t \sim$ $0.23 \mathrm{mag} \mathrm{day}^{-1}$, is reported in Figure 5. The range in color evolution is reminiscent of the two classes introduced by Stritzinger et al. (2018), with negative slopes consistent with their red class while flatter slopes with their blue class. When comparing data to models (Section 4) and when investigating the possible presence of multiple populations (Section 5), we will focus on the time evolution $\Delta(g-r) / \Delta t$ rather than the absolute values of colors. We consider this choice more robust as it is less sensitive to uncertainties introduced by both reddening corrections and $K$-corrections.

\section{Comparison to Models}

In this section, we compare the $g-r$ evolution of our sample with model predictions. In particular, we focus on three different scenarios that have been shown to produce characteristic signatures in the colors at early times (see discussion in Section 1). Specifically, we explore the SN ejecta-companion interaction model (Section 4.1), the double-detonation scenario (Section 4.2), and models with different amounts of ${ }^{56} \mathrm{Ni}$ mixed throughout the ejecta (Section 4.3). The peculiar "02cx-like," "Ia-CSM," and "super-Chandrasekhar" events are not considered in these comparisons.

We note that models presented here are plotted relative to explosion, while data are shown relative to first light $t_{\mathrm{fl}}$. Many of the SNe Ia in our sample (especially those at low redshift) are detected 4-5 mag below peak (Yao et al. 2019) and thus $t_{\mathrm{fl}}-t_{\mathrm{exp}}$ is expected to be small for these events according to predictions from explosion models $(\lesssim 2$ days, see, e.g., Figure 4 in Dessart et al. 2014). Nevertheless, given the issues with inferring $t_{\exp }$ from observations and with having a common definition of $t_{\text {fl }}$ across different models, we choose not to apply any shift to either models or data but caution against making a one-to-one comparison between them.

\subsection{SN Ejecta-companion Interaction}

Figure 6 compares our sample to $\mathrm{SN}$ ejecta-companion models from Kasen (2010). Predicted colors are shown for the four different companion-star models discussed in Kasen (2010), i.e., three MS stars with different masses $\left(1,2\right.$, and $\left.6 M_{\odot}\right)$ and a $1 M_{\odot}$ RG star. Luminosity and temperature for each model is estimated using Equations (22) and (25) in Kasen (2010) and assuming an ejecta velocity $v=10^{4} \mathrm{~km} \mathrm{~s}^{-1}$ and an effective opacity $\kappa_{\mathrm{e}}=0.2 \mathrm{~cm}^{2} \mathrm{~g}^{-1}$. Fluxes and corresponding $g-r$ colors are then estimated under a blackbody approximation.
Curves are shown only in, the first $\sim 5$ days since first light when the emission from the SN ejecta-companion interaction is expected to be dominant (see, e.g., Equation (23) in Kasen 2010; Maeda et al. 2018).

All the models investigated predict similar and relatively blue colors at first light, $g-r \sim-0.5 \mathrm{mag}$, which then become redder with time following the decrease in temperature (see Equation (25) in Kasen 2010). The transition from bluer to redder colors is characterized by $\Delta(g-r) / \Delta t \gtrsim 0.1$ mag day $^{-1}$, with a slower evolution in the case the companion is a RG or for increasing masses in the MS case. As shown in Figure 5 and summarized in Table 1, we see evidence for such a rapid transition in five events: ZTF 18abgxvra (SN 2018efb), ZTF 18abukmty (SN 2018lpz), ZTF 18abwnsoc (SN 2018lpr), ZTF 18abdefet (SN 2018dds) and ZTF 18abpaywm (SN 2018fne). However, the latter four events are characterized by relatively high photometric uncertainties (see Figure 5) while ZTF 18abgxvra shows a sign of red bump in the color evolution and might thus come from a double-detonation explosion (see Section 4.2 and right panel of Figure 7). Moreover, a good match in color slopes is found only with the $1 M_{\odot} \mathrm{RG}$ companion-star model, which predicts a very strong bump in both UV and optical light curves (see Figure 3 of Kasen 2010) that is not found in any of these five events.

Our calculations assume a perfect alignment between the exploding white dwarf, the companion star and the observer. As shown by Kasen (2010), the signature of the collision should be prominent $\sim 10 \%$ of the times for a favorable observer orientation near the perfect alignment. While we cannot exclude the presence of a companion star for each individual SN, the large size of our sample suggests we should see the effect of an interaction in $\sim$ six events. As a consequence, the fact that we do not see any clear evidence for a $\mathrm{SN}$ ejecta-companion interaction poses challenges to this scenario to explain the bulk of the SN Ia population.

\subsection{Helium-ignited Double-detonation Models}

Figure 7 shows the $g-r$ evolution of our $65 \mathrm{SNe}$ Ia compared to that predicted by helium-ignited double-detonation models from the literature. The left panel includes models from Polin et al. (2019a) with fixed helium mass $M_{\mathrm{He}}=0.01 M_{\odot}$ and varying carbon-oxygen core masses, while the right panel models from Noebauer et al. (2017) and Polin et al. (2019a) with carbon-oxygen core masses of $M_{\mathrm{CO}} \sim 1.0 M_{\odot}$ and varying helium shell masses in the range $M_{\mathrm{He}} \in[0.02,0.10] M_{\odot}$.

Models with very thin helium layers (left panel) show a range in the early-time color slopes. Models with $M_{\mathrm{CO}}=0.9$ and $1.0 M_{\odot}$ are characterized by steep transitions from red to bluer colors, while those with $M_{\mathrm{CO}}=1.1$ and $1.2 M_{\odot}$ by flatter evolutions. As shown in Figure 5, this range in slopes is in reasonable agreement with that observed in our ZTF sample although it cannot explain the events with $\Delta(g-r) / \Delta t \gtrsim 0$ mag day ${ }^{-1}$. We note that the four double-detonation models used here are those that have been claimed to explain maximum-light colors, velocity (Polin et al. 2019a), polarization (Cikota et al. 2019), and nebular calcium emission (Polin et al. 2019b) of a subset of SNe Ia. Our findings bring additional support to these claims, suggesting that the double-detonation scenario might contribute to some fraction of the observed SN Ia population. Specifically, the comparison in Figure 5 suggests that the double detonation models can explain the range in slopes observed for $\sim 60 \%$ (21 out of 34) of the events. 


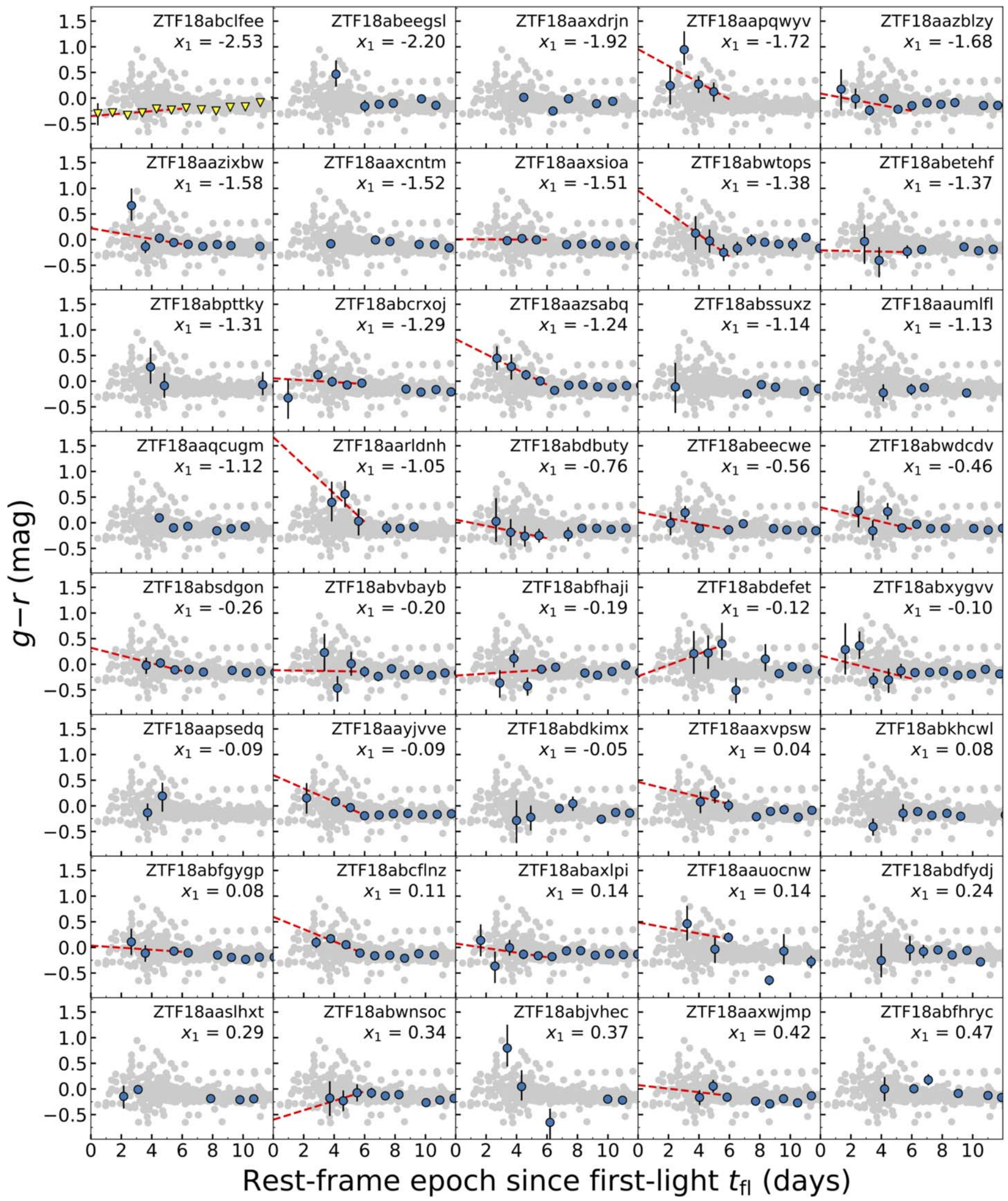

Figure 4. Same as Figure 1 but with the $g-r$ evolution of each of the 65 SNe highlighted. SNe are ordered from top-left to bottom-right according to their SALT2 $x_{1}$ values (Yao et al. 2019). Gray points mark the colors of the full sample for comparison. The red-dashed line in each panel is a weighted least-square linear fit to colors in the first 6 days for events with at least three data points in this time window. Colors and symbols are the same as in Figure 1. 


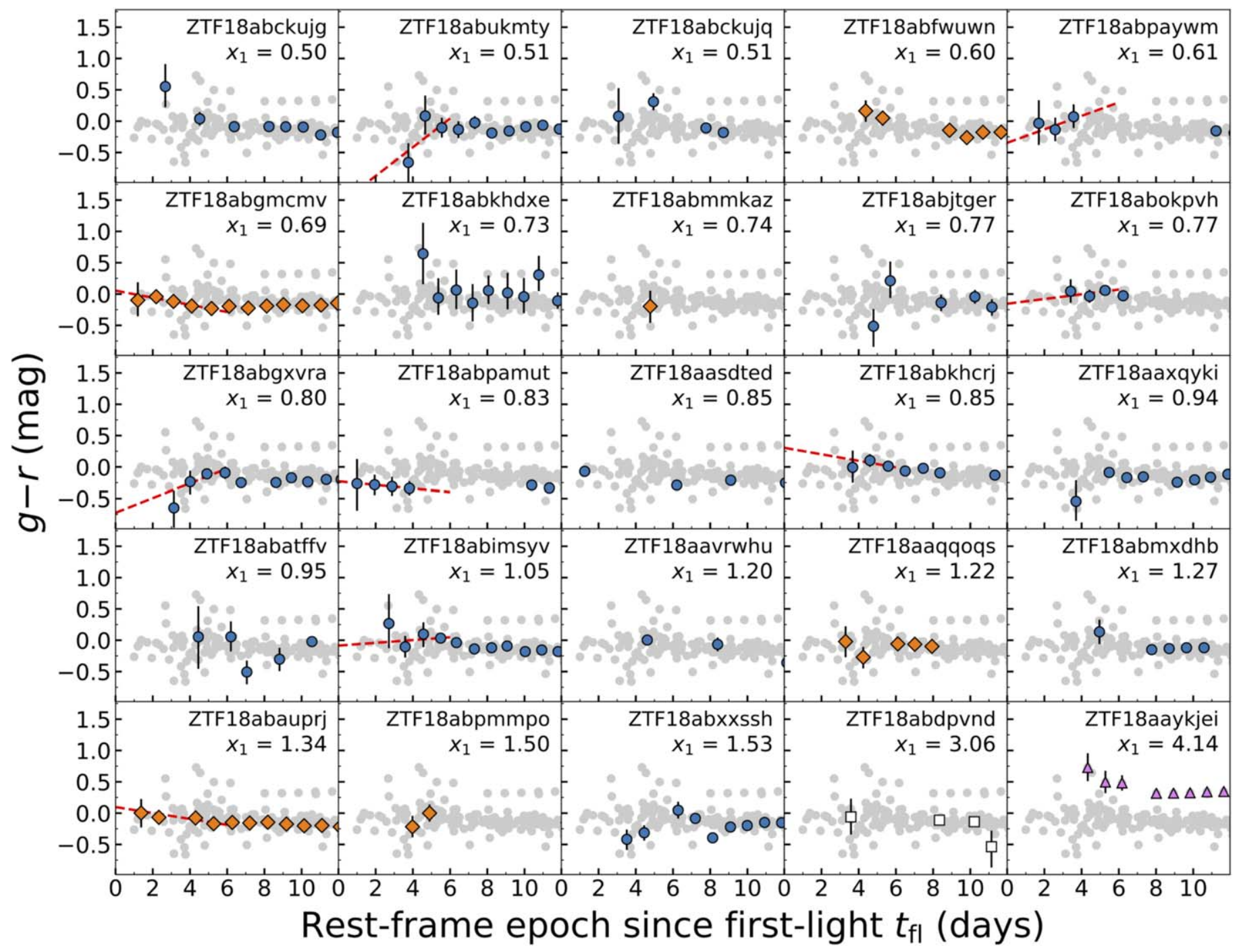

Figure 4. (Continued.)

Models with relatively thicker helium layers $\left(0.02 \lesssim M_{\mathrm{He}} \lesssim\right.$ $0.07 M_{\odot}$, right panel) produce strong red bumps (see Section 1). Visually inspecting the color evolution of each $\mathrm{SN}$ in Figure 4, we find evidence for a modest red bumps in six events: ZTF 18abcflnz (SN 2018cuw), ZTF 18abxxssh (SN 2018gvj), ZTF 18abcrxoj (SN 2018cvw), ZTF 18abgxvra (SN 2018efb), ZTF 18abckujq (SN 2018cvf), and ZTF 18aapqwyv (SN 2018bhc). All these six events display $g-r$ colors that are relatively blue at detection, ${ }^{20}$ evolve to redder colors, reach $g-r \sim 0$ at $\sim 3-6$ days after $t_{\mathrm{ff}}$ and then turn over to bluer colors. This temporal evolution is in good agreement with predictions from, e.g., Noebauer et al. (2017), suggesting that these $\mathrm{SNe}$ might come from double-detonation explosions of sub- $M_{\text {ch }}$ white dwarfs with relatively thick helium layers $\left(M_{\mathrm{He}} \sim 0.05 M_{\odot}\right)$. In addition, ZTF 18abxxssh (SN 2018gvj) is characterized by a strong light-curve excess at early times (Yao et al. 2019), making the interpretation of this SN within the double-detonation framework even more viable. The detection in six out of $65 \mathrm{SNe}$ suggests a red bump might occur in $\sim 9 \%$ of the cases. We note that these estimates are not representative

\footnotetext{
${ }^{20}$ We note that this statement relies somewhat on the rather large uncertainties in $g-r$ colors at detection.
}

of the double-detonation contribution to the SN Ia population, but rather of a subclass with relatively thick helium mass and thus detectable red bump. As discussed above, doubledetonation models with thin helium layers $\left(M_{\mathrm{He}}=0.01 M_{\odot}\right)$ might instead explain a good fraction $(\sim 60 \%)$ of the observed population.

\section{3. ${ }^{56} \mathrm{Ni}$ Mixing}

Figure 8 shows comparison between our sample and models exploring different amounts of ${ }^{56} \mathrm{Ni}$ mixing, where the color coding in all the different panels spans from red to blue for an increasing amount of mixing.

The top panel refers to models of Piro \& Morozova (2016), where mixing is implemented using a "boxcar" average with widths between 0.05 and $0.25 M_{\odot}$. As described in Section 1, models with stronger mixing are characterized by bluer colors at early times and relatively flatter evolution. Models by Piro \& Morozova (2016) are qualitatively in good agreement with our data, both in terms of colors and color evolution (see Figure 5). This comparison tentatively suggests that some amount of mixing is required to reproduce the average colors in the first few days after first light. We note, however, that local 


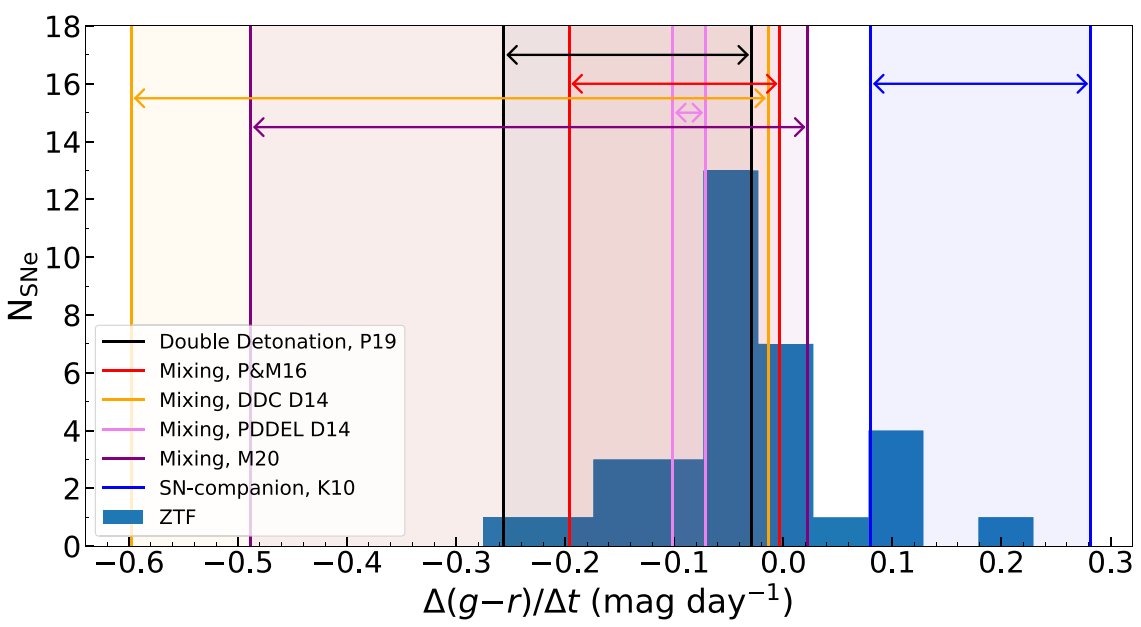

Figure 5. Comparison between observed and modeled slopes in the first 6 days since first light. The distribution refers to the linear slopes $\Delta(g-r) / \Delta t$ measured for $34 \mathrm{SNe}$ Ia with at least three detections in the first 6 days (see Section 3.2, the peculiar 02cx-like SN ZTF 18abclfee/SN 2018cxk is not considered here). The range spanned by each series of models is shown with a shaded area and with an horizontal arrow. Mixing models are from Piro \& Morozova (2016, red), Dessart et al. (2014, orange, and violet), and Magee et al. (2020, purple), with an increasing amount of mixing from left to right (vertical lines). The range spanned by the four SNcompanion interaction models from Kasen (2010) is shown in blue, while that from the double-detonation models of Polin et al. (2019a) with $M_{\mathrm{He}}=0.01 M_{\odot}$ in black. See text for more details.

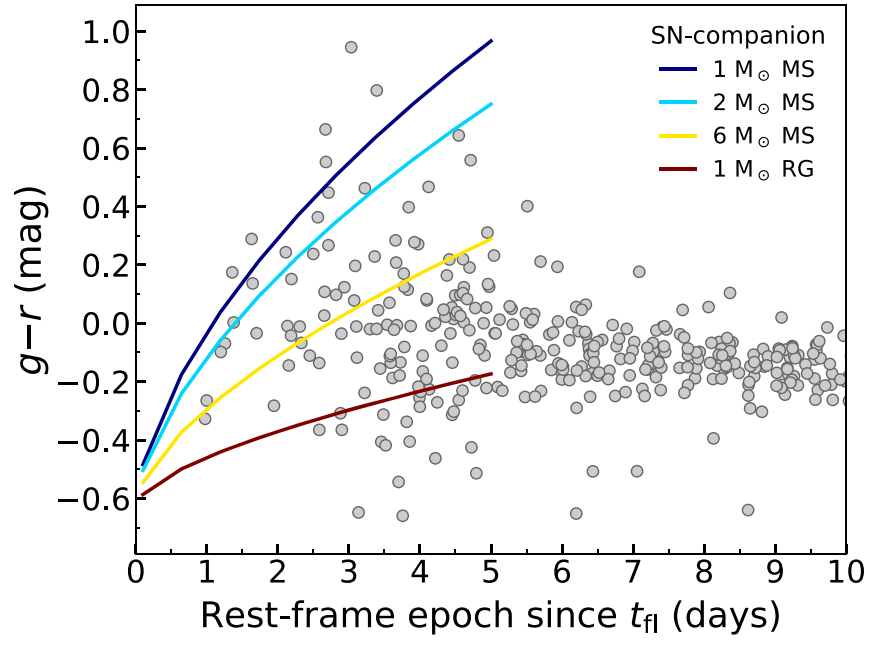

Figure 6. Comparison of our ZTF sample (gray points) to $\mathrm{SN}$ ejectacompanion models from Kasen (2010). Model predictions are shown for MS stars with three different masses $\left(1,2\right.$, and $\left.6 M_{\odot}\right)$ and for a $1 M_{\odot}$ RG star. Predicted colors are shown only in the first $\sim 5$ days since $t_{\mathrm{fl}}$ when the emission from the $\mathrm{SN}$ ejecta-companion interaction is expected to be dominant (see e.g., Equation (23) in Kasen 2010).

thermodynamic equilibrium (LTE) is assumed by Piro \& Morozova (2016) and thus predicted colors should be treated with caution.

The middle panel of Figure 8 shows comparison with models by Dessart et al. (2014) and a more recent (and unpublished) incarnation (DDC15m, this model was computed using the same approach as in Dessart et al. (2014) and differs only in the strength of mixing, as explained below). Unlike in Piro \& Morozova (2016), Dessart et al. (2014) carry out radiative transfer calculations for hydrodynamical models of $M_{\mathrm{ch}}$ delayed detonations (denoted as DDC10 and DDC15). All elements are mixed using a boxcar algorithm adopting a characteristic velocity $v_{\text {mix }}=250$ (DDC10_M1), 500 (DDC10_M2), 1000 (DDC10_M3), and $1500 \mathrm{~km} \mathrm{~s}^{-1}$ (DDC10_M4). We also include the delayed-detonation model DDC15 (Dessart et al. 2014), characterized by a relatively weak mixing of elements (model DDC $15 \mathrm{n} ; v_{\text {mix }}=400 \mathrm{~km} \mathrm{~s}^{-1}$ ). In contrast, the new unpublished model DDC15m is strongly mixed and similar to the most mixed of the Piro \& Morozova (2016) models (top panel of Figure 8). In model DDC $15 \mathrm{~m}$, the mixing is done using $m_{\text {mix }}=0.25 M_{\odot}$, together with a Gaussian smoothing with a characteristic width of $300 \mathrm{~km} \mathrm{~s}^{-1}$. These models also predict bluer and flatter colors for increasing amount of mixing, however, the colors in the first few days are relatively redder than those by Piro \& Morozova (2016). ${ }^{21}$ This is caused in part by the fact that the mixing in mass space pollutes the outer (high velocity) ejecta layers much more efficiently that mixing in velocity space. This arises because little mass is contained in the high velocity layers of the ejecta (in model DDC10, there is about $0.2 M_{\odot}$ beyond $15,000 \mathrm{~km} \mathrm{~s}^{-1}$ ). There may also be an opacity effect. Line blanketing below $5000 \AA$ remains strong out to large velocities well above the optical photosphere, so that the SN optical color is only set at large velocity. Guessing the $\mathrm{SN}$ color at the photosphere by inspecting the local LTE temperature is inaccurate and likely overestimates the true optical color. The strongly mixed model DDC $15 \mathrm{~m}$ is about 0.15 mag redder than the most mixed model from Piro \& Morozova, and appears somewhat too red relative to the observed mean $g-r$ color distribution (see also Dessart et al. 2014 and Miller et al. 2018). Although the colors are relatively redder than those observed, we note that the spread in slope predicted by the DDC10 and DDC15 suggests that some amount of mixing is required to explain the observed distribution shown in Figure 5.

Also included in the middle panel of Figure 8 are the PDDEL models of Dessart et al. (2014). The explosion mechanism in this scenario is similar to the delayed-detonation mechanism but here a delay is introduced between the initial deflagration and the subsequent detonation (Hoeflich \& Khokhlov 1996). This first pulse partially unbinds the outer layers of the $M_{\mathrm{ch}}$ white dwarf, so that the delayed detonation leads to a strong interaction between the detonated inner ejecta and the marginally unbound outer ejecta. The interaction leads to a strong dissipation of kinetic energy into heat, the formation of a dense shell at around 10,000-15,000 $\mathrm{km} \mathrm{s}^{-1}$, with little

\footnotetext{
$\overline{{ }^{21}}$ We note that the discrepancy could be reduced with a shift of $\sim 1-2$ days to account for the difference between $t_{\mathrm{fl}}$ and $t_{\mathrm{exp}}$, see above.
} 

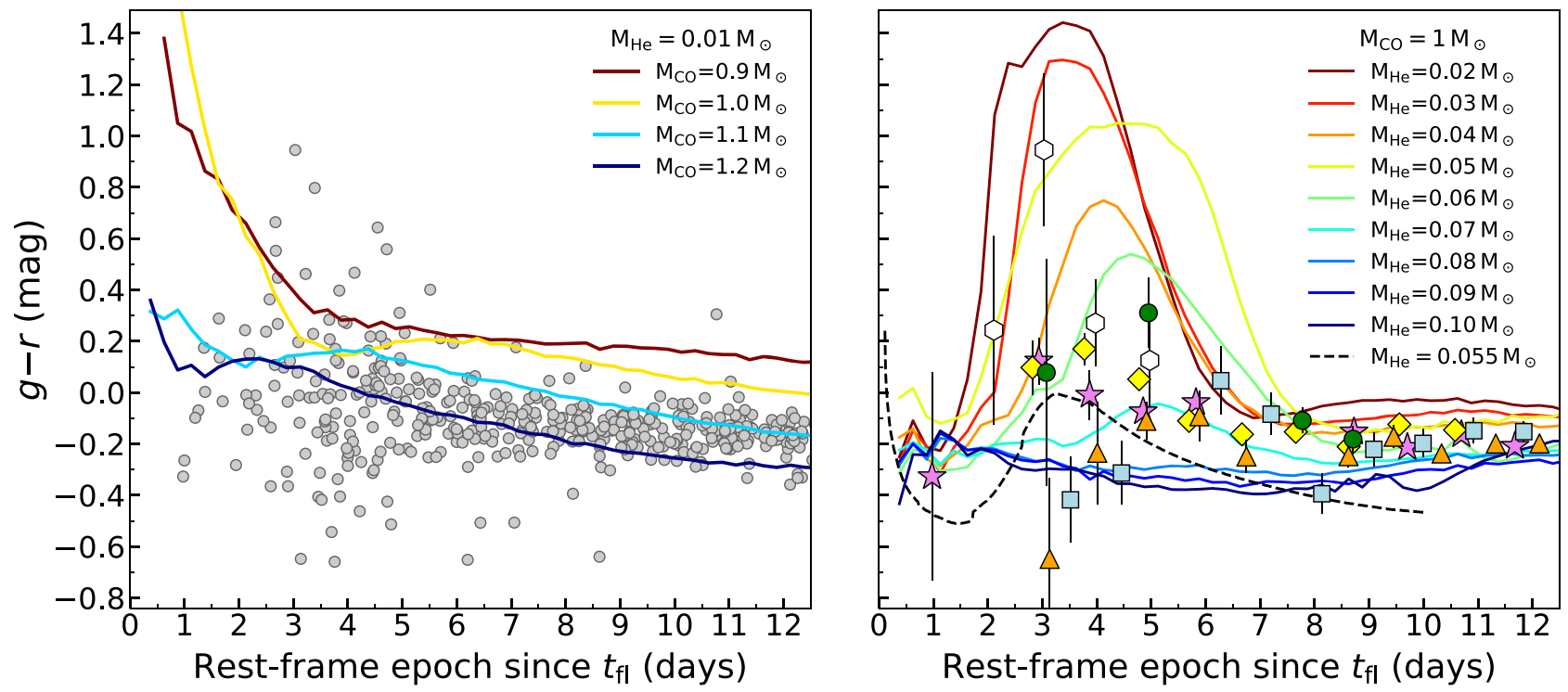

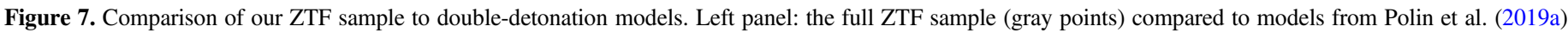

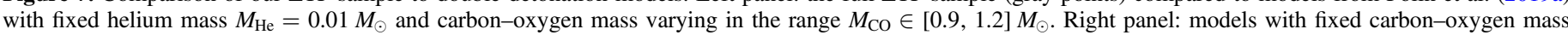

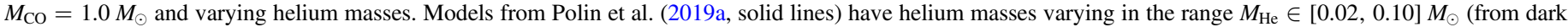

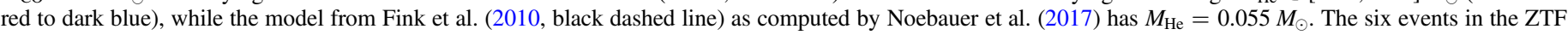

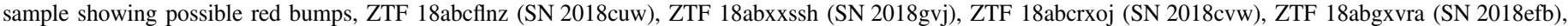

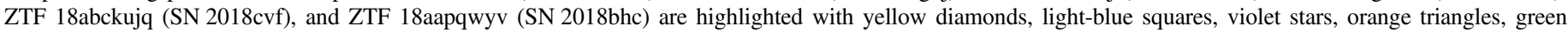
circles, and white hexagons, respectively.

mass beyond. ${ }^{22}$ Dessart et al. (2014) demonstrated that the early boost of the outer ejecta temperature had observable consequences for days on the luminosity and color, yielding brighter and bluer SNe. The models in Dessart et al. (2014) were however characterized by a weak mixing. Here, we recomputed the model PDDEL4 of Dessart et al. (2014) by using the same mixing recipe as for model DDC $15 \mathrm{~m}$ above. We refer to this model as PDDEL4m. For comparison, we include the weakly mixed model PDDEL4 (here called PDDEL4n) of Dessart et al. (2014). As can be seen from Figure 8, model PDDEL4m yields much bluer colors with a flatter evolution than the delayed-detonation model DDC15m (i.e., with no pulsation). Because of the red-to-blue transition predicted in the first $\sim 3$ days, this model struggles to reproduce the flatter-end of the observed $\Delta(g-r) / \Delta t$ distribution (see Figure 5).

The bottom panel of Figure 8 includes mixing models from Magee et al. (2020), computed using the radiative transfer code TURTLS (Magee et al. 2018). The grid of light-curve models is constructed with varying four main parameters: the ${ }^{56} \mathrm{Ni}$ mass $\left(0.4,0.6,0.8 M_{\odot}\right)$, the density profile shape (double power law or exponential), the kinetic energy, and the amount of ${ }^{56} \mathrm{Ni}$ mixing (see Magee et al. 2020 for more details). Here we compare our data to models producing $0.6 M_{\odot}$ of ${ }^{56} \mathrm{Ni}$ and for each mixing value plot the range covered by different density profiles and kinetic energy. The comparison highlights how the observed $g-r$ evolution is well reproduced by models requiring some degree of ${ }^{56} \mathrm{Ni}$ mixing (see Figure 5). In particular, the strongest agreement with data in the first 6 days is found for the "P100," "P21," "P9.7," and "P4.4" mixing models, with $\sim 67 \%$ of the data points falling in the color

\footnotetext{
$\overline{22}$ The pulsational-detonation scenario may correspond to an explosion configuration similar to the merging of two white dwarfs followed by a detonation. The marginally bound material from the pulsation in the PDDEL model corresponds now to the material that was flung during the merger and created a cocoon around the detonating residual.
}

range predicted by these models. We note that the more stratified models "P100" and "P21" were disfavored by Magee et al. (2020) based on comparisons to early light curves of normal SNe Ia.

Mixing is parameterized in all the models presented above and thus discrepancies with data do not necessarily rule out mixing scenarios but perhaps suggest that the mixing is different than adopted. Nevertheless, the range in slopes measured for our sample is in good agreement with the color evolution predicted by mixing models and better explained by incarnations requiring relatively strong ${ }^{56} \mathrm{Ni}$ mixing throughout the ejecta.

\section{Testing for Multiple Populations}

In this section, we take a closer look at the color evolution of $g-r$ colors at early phases, with the aim of testing the claim of two distinct populations made by Stritzinger et al. (2018, see Section 1). Specifically, we will base our discussion on $34 \mathrm{SNe}$ Ia with reliable $g-r$ slopes in the first 6 days $(\Delta(g-r) / \Delta t)$ as discussed in Section 3.2 (the peculiar 02cx-like SN ZTF 18abclfee/SN 2018cxk is excluded from this analysis). In particular, we will first test the presence of multiple populations in Section 5.1 and then search for possible correlations between color evolution and brightness in Section 5.2.

\subsection{Gaussian Mixture Models}

To test the claim of distinct populations, we apply Gaussian mixture models with single or multiple components to $\Delta(g-$ $r) / \Delta t$. In order to select how many components best fit the data, we use the Bayesian information criteria (BIC; Schwarz 1978) defined as

$$
\mathrm{BIC}=-2 \ln \mathcal{L}+k \ln N,
$$

where $\mathcal{L}$ is the maximum likelihood, $N$ the number of data points, and $k$ the number of parameters. The best model is the one with the lowest BIC, with the other models strongly disfavored if 
Weak mixing

Strong mixing
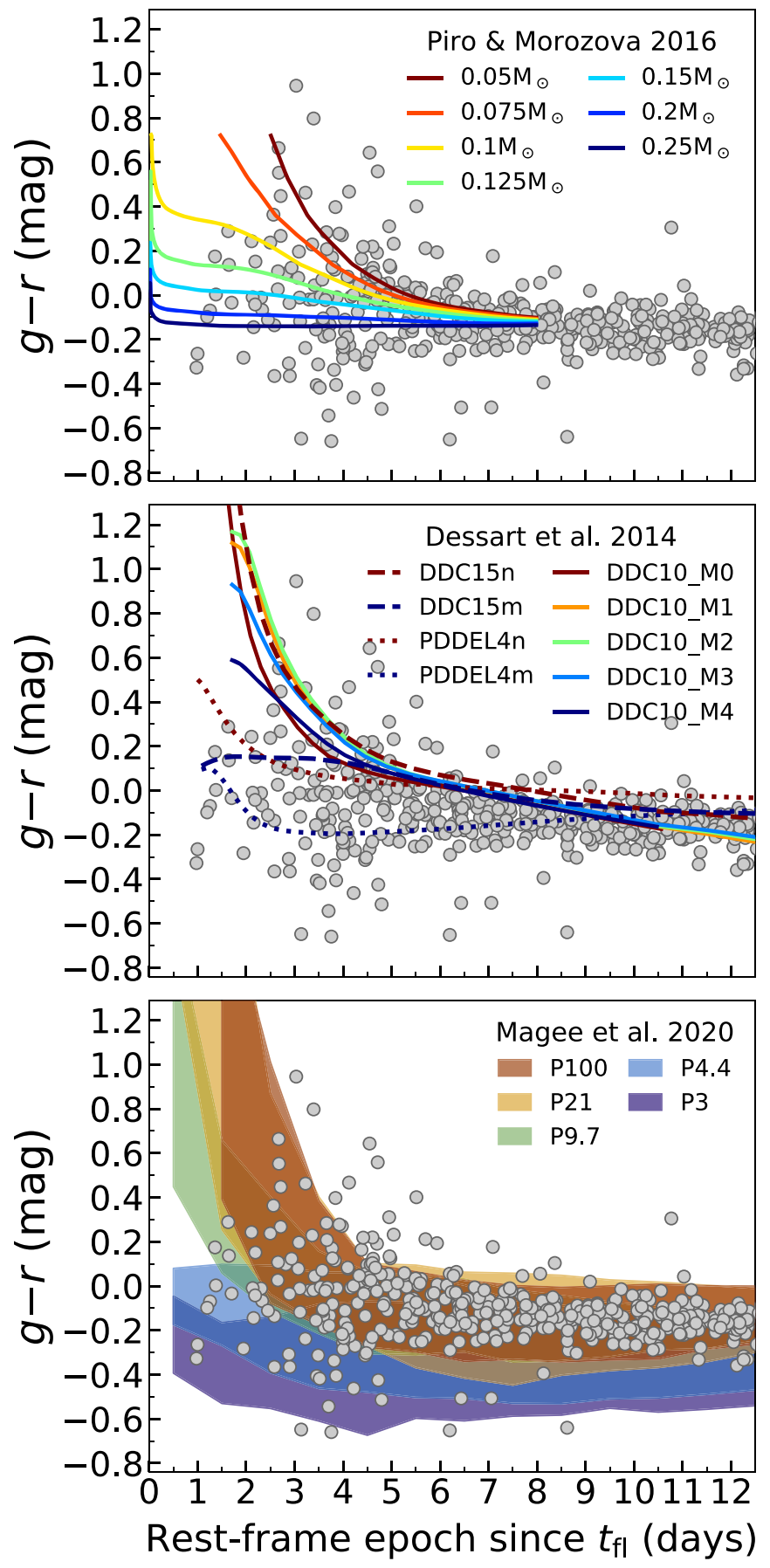

Figure 8. Comparison between our sample (gray points) and mixing models. The amount of mixing increases from models in red to models in blue. Top panel: models from Piro \& Morozova (2016). Middle panel: DDC (solid and dashed lines) and PDDEL (dotted lines) models from Dessart et al. (2014), together with the unpublished models DDC15m and PDDEL4m. Bottom panel: models from Magee et al. (2020) using the radiative transfer code TURTLS (Magee et al. 2018). For each mixing model, the shaded area represents color variations for different density profile shapes and kinetic energies (see text for details).

the difference to the best-fit model, $\Delta$ (BIC), is larger than 6 (see, e.g., Sollerman et al. 2009). The difference between different IC approaches lies in how much multiple-component models (and thus an added complexity) are penalized compared to a single-component model. As discussed in Liddle (2004), we choose BIC as this penalizes complexity/number of parameters more compared to, e.g., the Akaike information criteria (Akaike 1974).

Results of this analysis are summarized in the top panel of Figure 9, where we show the distribution of $\Delta(g-r) /$ $\Delta t$ values together with the BIC best-fit model. We find BIC $\sim-56.3,-46.6,-43.9$, and -36.4 for models with one, two, three, and four components, respectively. Therefore, the distribution is consistent with being drawn from one single population, i.e., $\min (\mathrm{BIC})=\mathrm{BIC}_{1 \mathrm{C}}$. In addition, a one component is not only preferred but strongly favored over multiple-component models $(\Delta \mathrm{BIC}>6)$.

To summarize, the color evolution in the first $\sim 6$ days after first light does not show any evidence for two or multiple components and it is consistent with being drawn from a single population. This conclusion is in contrast with the claim in Stritzinger et al. (2018) although we note that $B-V$ might be a better combination compared to $g-r$ to test for the presence of multiple populations (see Section 3.1). Our findings are consistent with the $B-V$ color evolution reported in (Han et al. 2020, see their Figure 5), where there appears to be no gap between the red and blue class when adding six events to the sample of Stritzinger et al. (2018). Surprisingly, Han et al. (2020) claims the presence of two distinct classes, although we note that similarly to Stritzinger et al. (2018) no analysis is provided to corroborate their conclusion.

\subsection{Color Evolution versus Brightness}

The bottom panel of Figure 9 show values of $\Delta(g-r) /$ $\Delta t$ against the SALT2 $x_{1}$ parameter, where the latter is used as a proxy for the SN brightness (with brighter events corresponding to larger $x_{1}$ ). We find a moderate correlation between the linear slope $\Delta(g-r) / \Delta t$ and SALT2 $x_{1}$. Specifically, the Pearson's correlation coefficient of $\rho=0.46$ suggests that this correlation is significant ( $p$-value of 0.006 , i.e., statistically significant at the significance level of 0.01). Relatively brighter events (large $x_{1}$ ) are preferentially associated with $g-r$ colors that are flat or evolving to redder colors, $\Delta(g-r) / \Delta t \gtrsim 0$. In contrast, relatively fainter events (small $x_{1}$ ) are characterized by colors becoming bluer with time, $\Delta(g-r) / \Delta t<0$. We have tested, and confirmed, that this correlation is present and statistically significant for different choices of the selection criterion, i.e., the correlation persists for cuts at 3, 3.5, 4, and 4.5 days since first light.

To summarize, we find a moderate correlation between brightness and color slope in the first $\sim 6$ days, with brighter events preferentially associated to flatter evolutions while fainter $\mathrm{SNe}$ are characterized by a transition from redder to bluer colors. These two behaviors are in qualitative agreement with those identified by Stritzinger et al. (2018) for their blue and red classes, respectively. However, our findings suggests that these are only the extremes of a continuous behavior, thus arguing against a bimodality (Stritzinger et al. 2018, see also Section 5.1).

\section{Discussion and Conclusions}

We presented $g-r$ colors for a sample of $65 \mathrm{SNe}$ Ia discovered within 5 days from first light by ZTF in 2018. The size of our sample is about three times larger than the one 
Best BIC model: 1 comp

$\mathrm{BIC}_{2 \mathrm{c}}-\mathrm{BIC}_{1 \mathrm{c}}=9.7$

$\mathrm{BIC}_{3 \mathrm{C}}-\mathrm{BIC}_{1 \mathrm{c}}=12.4$

$\mathrm{BIC}_{4 \mathrm{c}}-\mathrm{BIC}_{1 \mathrm{c}}=19.9$



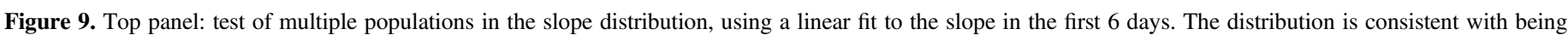

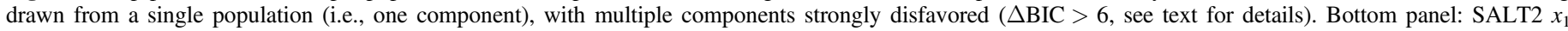

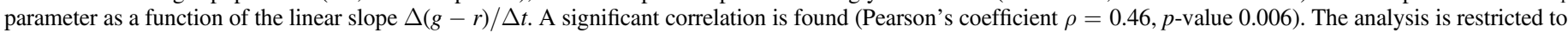

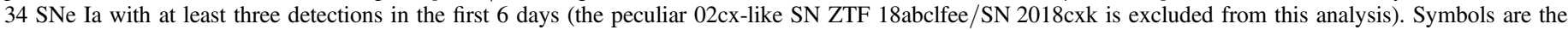
same as in Figure 1.

available in the literature and extends to higher redshifts (up to $z=0.143$ ). We find that $g-r$ colors are relatively homogeneous at all the phases investigated, from first light to $\sim 2$ weeks after. In particular, the observed scatter of $\sim 1.5 \mathrm{mag}$ at very early phases ( $\lesssim 6$ days) is roughly half intrinsic and half due to high photometric uncertainties. Specifically, we find that the intrinsic dispersion in $g-r$ colors in the first few days after explosion is smaller than that found in $B-V$ colors (Stritzinger et al. 2018) as a consequence of the different wavelength regions probed by different filter combination (Nordin et al. 2018).

We do, however, note different behaviors in the color evolution from first light to $\sim 6$ days later. In particular, some events have a rather steep change from redder to bluer colors while others are characterized by a flatter evolution. We further identify a significant correlation ( $\rho=0.46, p$-value of 0.006 ) between the SALT2 $x_{1}$ parameter and the linear color slope in the first 6 days, indicating that brighter events (large $x_{1}$ ) have flatter color evolutions at early times. However, contrary to previous claims in the literature (Stritzinger et al. 2018), the slope distribution does not show any evidence for bimodality and it is consistent with being drawn from a single population. We note that our findings are based on a subsample of 34 normal SNe Ia with at least three detections in the first 6 days since first light, a sample that is about twice (and not three times, see above) as large as the one in the literature after applying the same criteria (Stritzinger et al. 2018; Han et al. 2020).

The range in early-time slopes is reminiscent of mixing models, where an increasing amount of ${ }^{56} \mathrm{Ni}$ mixing in the outer ejecta regions leads to a transition from colors rapidly changing from red to blue to colors with a flatter evolution. In this context, the correlation found between early-time color slopes and brightness suggests that stronger mixing (hence flatter color evolution) might occur in explosions producing more ${ }^{56} \mathrm{Ni}$ (hence brighter). At the same time, the range in early-time slopes is in good agreement with predictions from helium-ignited double-detonation models with very thin helium layers $\left(M_{\mathrm{He}}=0.01 M_{\odot}\right)$ and varying carbon-oxygen masses between 0.9 and $1.2 M_{\odot}$ (Polin et al. 2019a). In addition, six $\mathrm{SNe}$ in our sample show evidence for a distinctive earlytime red bump predicted by double-detonation models with larger helium masses $\left(0.02 M_{\odot} \lesssim M_{\mathrm{He}} \lesssim 0.07 M_{\odot}\right.$, Noebauer et al. 2017; Polin et al. 2019a). Our findings support recent claims in the literature arguing that a subset of $\mathrm{SNe}$ Ia originates from double-detonation explosions (Cikota et al. 2019; Polin et al. 2019a, 2019b). In contrast, we find no clear evidence for a rapid transition from blue to red colors predicted by the ejecta-companion model discussed by Kasen (2010), 
posing serious challenges to this scenario for explaining the bulk of SNe Ia.

Based on the number of young SNe Ia discovered from 2018 May to December and presented here, the $3 \mathrm{yr}$ ZTF survey is expected to have a final sample of at least $\sim 200 \mathrm{SNe}$ Ia discovered within 5 days from first light. Such a large sample will allow us to place stronger constraints on explosion models and test the possible correlation between color evolution and brightness identified in this work.

The authors are thankful to Tony Piro for sharing his models, and to Chris Ashall, Joel Johansson, Mark Magee, Keiichi Maeda, and Stuart Sim for useful discussions.

M.B. acknowledges support from the G.R.E.A.T research environment funded by the Swedish National Science Foundation. A.A.M. is funded by the Large Synoptic Survey Telescope Corporation, the Brinson Foundation, and the Moore Foundation in support of the LSSTC Data Science Fellowship Program; he also receives support as a CIERA Fellow by the CIERA Postdoctoral Fellowship Program (Center for Interdisciplinary Exploration and Research in Astrophysics, Northwestern University). This research was supported in part through the computational resources and staff contributions provided for the Quest high performance computing facility at Northwestern University which is jointly supported by the Office of the Provost, the Office for Research, and Northwestern University Information Technology. This work was supported in part by the GROWTH project funded by the National Science Foundation under grant No. 1545949. SRK thanks the Heising-Simons Foundation for supporting his ZTF research.

This work is based on observations obtained with the Samuel Oschin Telescope 48 inch and the 60 inch Telescope at the Palomar Observatory as part of the Zwicky Transient Facility project. ZTF is supported by the National Science Foundation under grant No. AST-1440341 and a collaboration including Caltech, IPAC, the Weizmann Institute for Science, the Oskar Klein Center at Stockholm University, the University of Maryland, the University of Washington, Deutsches Elektronen-Synchrotron, and Humboldt University, Los Alamos National Laboratories, the TANGO Consortium of Taiwan, the University of Wisconsin at Milwaukee, and Lawrence Berkeley National Laboratories. This work was supported by the GROWTH project (Kasliwal et al. 2019) funded by the National Science Foundation under grant No. 1545949. Operations are conducted by COO, IPAC, and UW. This work made use of the Heidelberg Supernova Model Archive (HESMA), https://hesma.h-its.org.

\section{ORCID iDs}

Mattia Bulla (ib https://orcid.org/0000-0002-8255-5127 Adam A. Miller (1) https://orcid.org/0000-0001-9515-478X Yuhan Yao (10) https://orcid.org/0000-0001-6747-8509 Luc Dessart (i) https://orcid.org/0000-0003-0599-8407 Suhail Dhawan (1) https://orcid.org/0000-0002-2376-6979 Semeli Papadogiannakis (i) https://orcid.org/0000-00030783-3323

Rahul Biswas (D) https://orcid.org/0000-0002-5741-7195 Ariel Goobar (1) https://orcid.org/0000-0002-4163-4996 S. R. Kulkarni (1) https://orcid.org/0000-0001-5390-8563 Peter Nugent (iD https://orcid.org/0000-0002-3389-0586 Abigail Polin (iD https://orcid.org/0000-0002-1633-6495
Jesper Sollerman (1D https://orcid.org/0000-0003-1546-6615

Eric C. Bellm (1D https://orcid.org/0000-0001-8018-5348

Michael W. Coughlin (10) https://orcid.org/0000-00028262-2924

Richard Dekany (10) https://orcid.org/0000-0002-5884-7867

V. Zach Golkhou (i) https://orcid.org/0000-0001-8205-2506

Matthew J. Graham (iD https://orcid.org/0000-0002-3168-0139

Mansi M. Kasliwal (iD https://orcid.org/0000-0002-5619-4938

Thomas Kupfer (iD https://orcid.org/0000-0002-6540-1484

Russ R. Laher (ib https://orcid.org/0000-0003-2451-5482

Frank J. Masci (ib https://orcid.org/0000-0002-8532-9395

Ben Rusholme (ib https://orcid.org/0000-0001-7648-4142

David L. Shupe (i) https://orcid.org/0000-0003-4401-0430

\section{References}

Akaike, H. 1974, ITAC, 19, 716

Bellm, E. C., Kulkarni, S. R., Graham, M. J., et al. 2019, PASP, 131, 018002

Branch, D., Dang, L. C., Hall, N., et al. 2006, PASP, 118, 560

Burns, C. R., Parent, E., Phillips, M. M., et al. 2018, ApJ, 869, 56

Burns, C. R., Stritzinger, M., Phillips, M. M., et al. 2014, ApJ, 789, 32

Cikota, A., Patat, F., Wang, L., et al. 2019, MNRAS, 490, 578

De, K., Kasliwal, M. M., Polin, A., et al. 2019, ApJL, 873, L18

Dessart, L., Blondin, S., Hillier, D. J., \& Khokhlov, A. 2014, MNRAS, 441, 532

Dimitriadis, G., Foley, R. J., Rest, A., et al. 2019, ApJL, 870, L1

Fink, M., Röpke, F. K., Hillebrandt, W., et al. 2010, A\&A, 514, A53

Foley, R. J., Challis, P. J., Filippenko, A. V., et al. 2012, ApJ, 744, 38

Graham, M. J., Kulkarni, S. R., Bellm, E. C., et al. 2019, PASP, 131, 078001

Graham, M. L., Foley, R. J., Zheng, W., et al. 2015, MNRAS, 446, 2073

Graham, M. L., Kumar, S., Hosseinzadeh, G., et al. 2017, MNRAS, 472, 3437

Han, X., Zheng, W., Stahl, B. E., et al. 2020, ApJ, 892, 142

Hoeflich, P., \& Khokhlov, A. 1996, ApJ, 457, 500

Hosseinzadeh, G., Sand, D. J., Valenti, S., et al. 2017, ApJL, 845, L11

Hsiao, E. Y., Burns, C. R., Contreras, C., et al. 2015, A\&A, 578, A9

Hsiao, E. Y., Conley, A., Howell, D. A., et al. 2007, ApJ, 663, 1187

Iben, I. J., \& Tutukov, A. V. 1984, ApJS, 54, 335

Jacobson-Galan, W. V., Polin, A., Foley, R. J., et al. 2020, ApJ, 896, 165

Jeffery, D. J., Leibundgut, B., Kirshner, R. P., et al. 1992, ApJ, 397, 304

Jiang, J.-A., Doi, M., Maeda, K., et al. 2017, Natur, 550, 80

Jiang, J.-a., Doi, M., Maeda, K., \& Shigeyama, T. 2018, ApJ, 865, 149

Kasen, D. 2010, ApJ, 708, 1025

Kasliwal, M. M., Cannella, C., Bagdasaryan, A., et al. 2019, PASP, 131, 038003

Li, W., Wang, X., Vinkó, J., et al. 2019, ApJ, 870, 12

Liddle, A. R. 2004, MNRAS, 351, L49

Livio, M., \& Mazzali, P. 2018, PhR, 736, 1

Livne, E. 1990, ApJL, 354, L53

Maeda, K., Jiang, J.-a., Shigeyama, T., \& Doi, M. 2018, ApJ, 861, 78

Magee, M. R., Maguire, K., Kotak, R., et al. 2020, A\&A, 634, A37

Magee, M. R., Sim, S. A., Kotak, R., \& Kerzendorf, W. E. 2018, A\&A, 614, A115

Masci, F. J., Laher, R. R., Rusholme, B., et al. 2019, PASP, 131, 018003

Mazzali, P. A., Danziger, I. J., \& Turatto, M. 1995, A\&A, 297, 509

Miller, A. A., Cao, Y., Piro, A. L., et al. 2018, ApJ, 852, 100

Miller, A. A., Yao, Y., Bulla, M., et al. 2020, arXiv:2001.00598

Moll, R., \& Woosley, S. E. 2013, ApJ, 774, 137

Noebauer, U. M., Kromer, M., Taubenberger, S., et al. 2017, MNRAS, 472, 2787

Nomoto, K. 1980, SSRv, 27, 563

Nordin, J., Aldering, G., Antilogus, P., et al. 2018, A\&A, 614, A71

Nugent, P. E., Sullivan, M., Cenko, S. B., et al. 2011, Natur, 480, 344

Papadogiannakis, S., Goobar, A., Amanullah, R., et al. 2019, MNRAS, 483, 5045

Piro, A. L., \& Morozova, V. S. 2016, ApJ, 826, 96

Polin, A., Nugent, P., \& Kasen, D. 2019a, ApJ, 873, 84

Polin, A., Nugent, P., \& Kasen, D. 2019b, arXiv:1910.12434

Riess, A. G., Filippenko, A. V., Li, W., et al. 1999, AJ, 118, 2675

Ruiz-Lapuente, P., Cappellaro, E., Turatto, M., et al. 1992, ApJL, 387, L33

Schwarz, U. J. 1978, A\&A, 65, 345

Shappee, B. J., Holoien, T. W. S., Drout, M. R., et al. 2019, ApJ, 870, 13 
Shappee, B. J., Piro, A. L., Holoien, T. W.-S., et al. 2016, ApJ, 826, 144

Silverman, J. M., Ganeshalingam, M., Cenko, S. B., et al. 2012, ApJL, 756, L7

Sollerman, J., Mörtsell, E., Davis, T. M., et al. 2009, ApJ, 703, 1374

Spiegelhalter, D. J., Best, N. G., Carlin, B. P., \& van der Linde, A. 2002, J. Royal Stat. Soc.: B (Statistical Methodology), 64, 583

Stritzinger, M. D., Shappee, B. J., Piro, A. L., et al. 2018, ApJL, 864, L35
Taam, R. E. 1980, ApJ, 237, 142

Vallely, P. J., Fausnaugh, M., Jha, S. W., et al. 2019, MNRAS, 487, 2372

Vinkó, J., Ordasi, A., Szalai, T., et al. 2018, PASP, 130, 064101

Webbink, R. F. 1984, ApJ, 277, 355

Whelan, J., \& Iben, I. J. 1973, ApJ, 186, 1007

Yao, Y., Miller, A. A., Kulkarni, S. R., et al. 2019, ApJ, 886, 152 